Pacific Journal of Mathematics

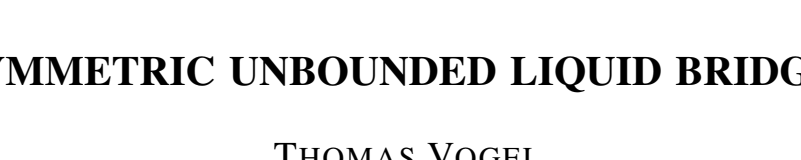




\title{
SYMMETRIC UNBOUNDED LIQUID BRIDGES
}

\author{
Thomas I. VOGEL
}

A symmetric unbounded liquid bridge is that surface formed by capillary action when a disc is withdrawn from an infinite reservoir. As do all capillary surfaces, liquid bridges satisfy the condition that mean curvature is proportional to height. The profile curves of the bridge surfaces are parametric extensions of the curves obtained in the symmetric exterior capillary problem. The family of profile curves is shown to have an envelope, and stability and instability criteria are derived relating to this envelope.

Introduction. An unbounded liquid bridge is that surface which is formed when an object is withdrawn a small distance from an infinite pool of liquid, and the liquid is allowed to reach an equilibrium (see Figure 1). The adjective "unbounded" serves to distinguish this surface from the bounded liquid bridges formed by drops of liquid between two parallel plates, or by an object withdrawn from a finite reservoir, neither of which will be studied in this paper.

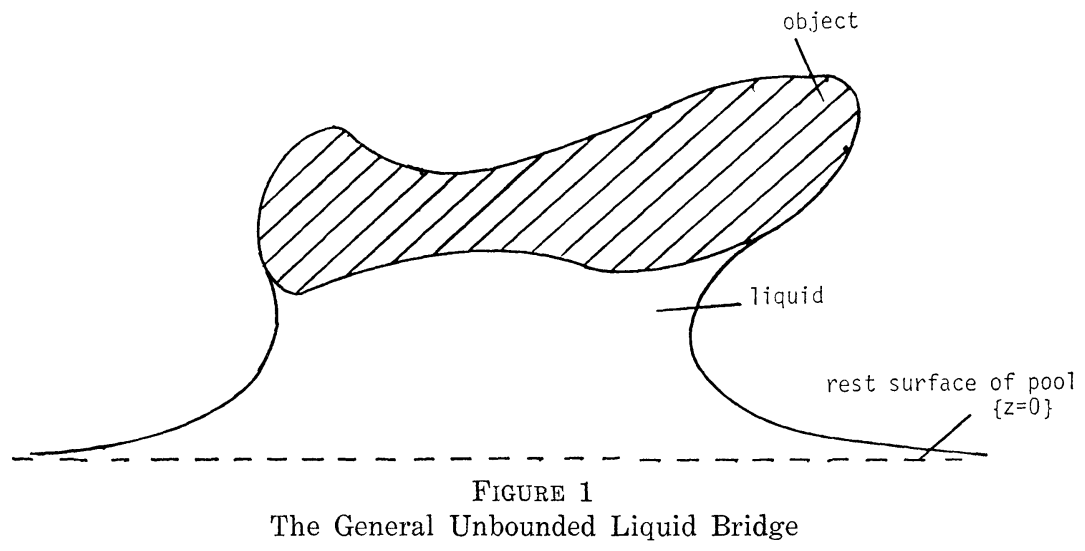

In this paper, I will deal only with the symmetric surface formed in three dimensions when the object withdrawn is a disc parallel to the base plane $\{z=0\}$. This is a problem in capillarity, so that the shape of the free surface is determined by the interaction of the potential energy due to the lifting of the liquid in a gravitational field, the surface tension of the liquid, and the energy gained by wetting the disc. Specifically, for a given compact set $K$, define an energy functional acting on sets by:

$$
\mathscr{E}_{K}\left(A^{\prime}\right) \equiv \sigma P\left(A^{\prime}, K\right)+\rho g \int_{A^{\prime} \cap K} z-\sigma \beta P\left(A^{\prime}, \mathscr{D} \cap K\right) .
$$


Here $\mathscr{D}$ is the disc lifted from the liquid, $P\left(A^{\prime}, K\right)$ is (roughly) the surface area of $\partial A^{\prime} \cap K, P\left(A^{\prime}, \mathscr{D} \cap K\right)$ is the area of $\partial A^{\prime} \cap \mathscr{D} \cap K$, $\sigma$ is the surface tension of the liquid, $\rho$ is its density, $g$ is the acceleration due to gravity, and $\beta$ is a constant depending on the liquid and the material of the disc. A set $A$ (corresponding to the liquid) will be a liquid bridge if for every compact set $K$ and every set $A^{\prime}$ equal to $A$ outside of $K$, we have that

$$
\mathscr{E}_{K}(A) \leqq \mathscr{E}_{K}\left(A^{\prime}\right)
$$

In $\S 1$, I show that a stable symmetric unbounded liquid bridge must wet the entire disc.

The Euler equation resulting from this functional requires that the mean curvature of $\partial A$ be proportional to height above the base plane. If in addition axial symmetry is assumed, then I obtain a system of O.D.E.'s describing the profile of $\partial A$. Sections 2, 3, and 4 of the present work go into detail in characterizing the profile curves. Of particular use is the fact that the family of profile curves has an envelope. This envelope is indispensable in the study of the conditions under which a given profile curve represents a stable unbounded liquid bridge (§5). Unfortunately, I have been unable to prove that the envelope is a $C^{1}$ curve, and have been forced to assume this (Conjecture 4.7) to obtain my stability and instability results. The only previous stability result I have been able to find is in a paper by Pitts [11]. He derives an instability criterion which seems equivalent to mine. However, he was forced to consider the slightly different problem of a large but finite pool with the pressure prescribed at the horizontal point of the bridge.

It is important to realize that $\partial A$ cannot be expected to be a graph over the reference plane $\{z=0\}$, that is, $\partial A$ could fold over. The unbounded liquid bridge problem is therefore in general a parametric capillary problem as are the pendent drop (see Wente [15], Concus \& Finn [3]), and the sessile drop for contact angle $>\pi / 2$ (see Gonzalez [6], [7], and Finn [4]).

1. An instability theorem. I will show that a symmetric unbounded liquid bridge cannot exist unless the entire disc is wetted. This has the curious effect of rendering the constant $\beta$ in (0.1) much less important in the characterization of liquid bridges than in other problems such as sessile and pendent drops. If two discs are the same height and have the same radius, and if both of them form nontrivial symmetric liquid bridges, then the bridges will be the same even if the values of $\beta$ are different. The value of $\beta$ will enter into stability considerations. 
The following calculation was suggested by Nicholas Korevaar:

THEOREM 1.1. The symmetric unbounded liquid bridge problem can only have a stable solution if the entire disc is wetted or none of it is (in which case the solution is trivial).

Proof. The proof proceeds by contradiction. Let $r=r(u)$ be the radius of the symmetric bridge at height $u$. If there is a stable symmetric bridge, then it is a fortiori stable under symmetric perturbations. I will show later that a bridge which is stable under symmetric perturbations is in fact stable under asymmetric perturbations. To solve the symmetric unbounded liquid bridge problem, therefore, is to find a smooth function $r$ which minimizes the following energy functional over compact perturbations:

$$
G(f)=\int_{0}^{h}\left[2 \sigma \pi f \sqrt{1+\left(f^{\prime}\right)^{2}}+\pi \rho g u f^{2}\right] d u-\pi \sigma \beta f^{2}(h),
$$

over the family of functions $f$ with $\lim _{u \rightarrow 0^{+}} f(u)=+\infty$. Here $h$ is the height of the disc above the reference plane $\{z=0\}$. If $r(h)$ is not equal to the radius of the disc, then it can vary in both directions. This fact will be used to construct a particular perturbation for which the second variation $\delta^{2} G(r)<0$, which will contradict the assumption that $r(u)$ is an energy minimum. To be precise, if, for $\eta>0$, I define:

$$
G_{\eta}(f)=\int_{\eta}^{h}\left[2 \pi \sigma f \sqrt{1+\left(f^{\prime}\right)^{2}}+\pi \rho g u f^{2}\right] d u-\pi \beta \sigma f^{\prime}(h),
$$

then I will attempt to find a function $y$ with $y(\eta)=0$ and

$$
\frac{d^{2}}{d \varepsilon^{2}}\left(G_{\gamma}(r+\varepsilon y)\right)<0
$$

at $\varepsilon=0$.

Writing everything out explicitly, I have:

$$
\begin{aligned}
\left.\frac{d G_{\eta}}{d \varepsilon}(r+\varepsilon y)\right|_{\varepsilon=0}= & 2 \pi \sigma \int_{\eta}^{h} y\left(\frac{1}{V 1+\left(r^{\prime}\right)^{2}}-\frac{r^{\prime \prime}}{\left(1+\left(r^{\prime}\right)^{2}\right)^{3 / 2}}+\kappa u r\right) d u \\
& +2 \pi \sigma r(h) y(h)\left[\frac{r^{\prime}(h)}{\sqrt{1+\left(r^{\prime}(h)\right)^{2}}}-\beta\right],
\end{aligned}
$$

where $k=(\rho g / \sigma)>0$ is the so-called "capillary constant". Suppose that $r(u)$ is a local minimum of $G_{\eta}$. From the above equation, I conclude:

$$
\frac{r^{\prime \prime}}{\left(1+\left(r^{\prime}\right)^{2}\right)^{3 / 2}}-\frac{1}{\sqrt{1+\left(r^{\prime}\right)^{2}}}=\kappa u r
$$


and

$$
\frac{r^{\prime}(h)}{\sqrt{1+\left(r^{\prime}(h)\right)^{2}}}=\beta \text {. }
$$

The latter equation comes from the fact that I allow $y(h)$ to be nonzero, i.e., that $r(u)$ is assumed to be a local minimum of $G_{\eta}$ over perturbations which leave $r(\eta)$ fixed, but which might change the value at $h$.

To find the second variation, I differentiate $G_{\eta}(\boldsymbol{r}+\varepsilon y)$ again to obtain:

$$
\begin{aligned}
\left.\frac{d^{2} G_{\eta}(r+\varepsilon y)}{d \varepsilon^{2}}\right|_{\varepsilon=0}= & 2 \pi \sigma \int_{\eta}^{h}\left(y^{\prime}\right)^{2}\left(\frac{r}{\left(1+\left(r^{\prime}\right)^{2}\right)^{3 / 2}}\right) \\
& +y^{2}\left(\kappa u-\frac{r^{\prime \prime}}{\left(1+\left(r^{\prime}\right)^{2}\right)^{3 / 2}}\right) d u,
\end{aligned}
$$

where the boundary term cancels out when $r$ satisfies (1.2). From (1.1) I may write:

$$
\left.\frac{d^{2} G_{\eta}(r+\varepsilon y)}{d \varepsilon^{2}}\right|_{\varepsilon=0}=2 \pi \sigma \int_{\eta}^{h}\left(y^{\prime}\right)^{2}\left(\frac{r}{\left(1+\left(r^{\prime}\right)^{2}\right)^{3 / 2}}\right)-y^{2}\left(\frac{1}{r \sqrt{1+\left(r^{\prime}\right)^{2}}}\right) d u
$$

I will produce a particular $y$ for which (1.3) is less than zero, thus proving $r(u)$ is unstable. Noticing that the coefficient of $y^{2}$ in (1.3) is negative, the natural plan to follow is to find a $y$ with $\int_{\eta}^{h}\left(y^{\prime}\right)^{2}\left(r /\left(1+\left(r^{\prime}\right)^{2}\right)^{3 / 2}\right) d u$ neglible compared to $\int_{\eta}^{h} y^{2}\left(1 / \boldsymbol{r} \sqrt{1+\left(\boldsymbol{r}^{\prime}\right)^{2}}\right) d u$, where $\eta$ is properly chosen. To do this, I need some idea of how fast $r$ and $r^{\prime}$ go to infinity as $u$ goes to zero, just using the fact that $r$ satisfies (1.1) and the condition that $\lim _{u \rightarrow 0} r(u)=+\infty$.

From Siegel's asymptotic estimates for the exterior capillary problem ([13]), I know that $u(r) \sim\left(C e^{-r} / \sqrt{r}\right)$ as $r$ goes to infinity, where $u(r)$ is the inverse function of $r(u)$ for small $u$, so that $u(r)>e^{-2 r}$ for sufficiently large $r$. It follows that $r(u)>-(1 / 2) \log u$ for $u$ sufficiently small, since both $u(r)$ and $e^{-2 r}$ are decreasing in $r$. It cannot be true that $\lim _{u \rightarrow 0}\left(r^{\prime}(u) /-(1 / 2 u)\right)=0$, or else by L'Hôpital's rule I would have $\lim _{u \rightarrow 0}(r(u) /-(1 / 2) \log u)=0$, which is absurd by the above. Hence there exists an $a>0$ and a sequence $\left\{u_{i}\right\}$ approaching zero with:

$$
\begin{aligned}
& \frac{r^{\prime}\left(u_{i}\right)}{\left(-\frac{1}{2 u_{i}}\right)}>a, \text { so } \\
& \left|r^{\prime}\left(u_{i}\right)\right|>\frac{a}{2 u_{i}},
\end{aligned}
$$


since $r^{\prime}(u)$ is negative for $u$ sufficiently small.

Define the perturbation $y_{i}(u)$ by:

$$
y_{i}(u)= \begin{cases}2 u / u_{i}-1 & u_{i} / 2 \leqq u \leqq u_{i} \\ 1 & u_{i} \leqq u \leqq h\end{cases}
$$

Then,

$$
\begin{aligned}
\int_{u_{i} / 2}^{h}\left(y_{i}^{\prime}(u)\right)^{2} \frac{r}{\left(1+\left(r^{\prime}\right)^{2}\right)^{3 / 2}} d u & =\int_{u_{i} / 2}^{u_{i}} \frac{4}{\left(u_{i}\right)^{2}} \frac{r}{\left(1+\left(r^{\prime}\right)^{2}\right)^{3 / 2}} d u \\
& \leqq \frac{4}{\left(u_{i}\right)^{2}} \int_{u_{i} / 2}^{u_{i}} \frac{r}{\left|\boldsymbol{r}^{\prime}\right|^{3}} d u .
\end{aligned}
$$

I now claim that $\lim _{u \rightarrow 0^{+}}\left(r^{\prime}(u) / r(u)\right)$ exists and equals $-\infty$. For its existence, note that:

$$
\frac{d}{d u}\left(\frac{r^{\prime}(u)}{r(u)}\right)=\frac{r r^{\prime \prime}-\left(r^{\prime}\right)^{2}}{r^{2}}=\frac{1+\kappa u r\left(1+\left(r^{\prime}\right)^{2}\right)^{3 / 2}}{r^{2}}>0
$$

from equation (1.1), so that $r^{\prime}(u) / r(u)$ is strictly increasing. That the limit must equal $-\infty$ is clear, since $r^{\prime}(u) / r(u)=d / d u(\log (r(u))$, where $\log r(u) \rightarrow+\infty$ as $u$ goes to zero. From this fact and equation (1.4), I may conclude that:

$$
\int_{u_{i} / 2}^{u_{i}}\left[y_{i}^{\prime}(u)\right]^{2} \frac{r}{\left(1+\left(r^{\prime}\right)^{2}\right)^{3 / 2}} d u \leqq \frac{4}{\left(u_{i}\right)^{2}} \int_{u_{i} / 2}^{u_{i}} \frac{1}{\left(r^{\prime}\right)^{2}} d u
$$

for sufficiently small $u_{i}$. Since $r^{\prime \prime}(u)>0, r^{\prime}$ is monotone, so for small $u$ I can estimate (1.5) by:

$$
\begin{aligned}
\int_{u_{i} / 2}^{u_{i}}\left[y_{i}^{\prime}(u)\right]^{2} \frac{r}{\left(1+\left(r^{\prime}\right)^{2}\right)^{3 / 2}} & \leqq \frac{4}{\left(u_{i}\right)^{2}}\left(\frac{1}{\left(r^{\prime}\left(u_{i}\right)\right)^{2}}\right) \cdot \frac{u_{i}}{2} \\
& <\frac{4}{\left(u_{i}\right)^{2}} \cdot \frac{4\left(u_{i}\right)^{2}}{a^{2}} \cdot \frac{u_{i}}{2}=\frac{8 u_{i}}{a^{2}},
\end{aligned}
$$

so as $i$ approaches infinity, this term goes to zero.

However, the other term in (1.3) is

$$
\int_{u_{i} / 2}^{h}\left[y_{i}(u)\right]^{2}\left(\frac{-1}{r\left(1+\left(r^{\prime}\right)^{2}\right)^{1 / 2}}\right) d u \leqq \int_{u_{i}}^{h} \frac{-1}{r\left(1+\left(r^{\prime}\right)^{2}\right)^{1 / 2}} d u,
$$

which stays strictly below zero as $i \rightarrow \infty$. This shows that $\left(d^{2} / d \varepsilon^{2}\right) G_{u_{i} / 2}\left(r+\varepsilon y_{i}\right)_{\varepsilon=0}<0$ for $i$ large enough, thus demonstrating that the symmetric liquid bridge problem has no stable solution if the endpoint $r(h)$ is not somehow restricted. The crucial point in this calculation is equation (1.2), which causes the boundary term to cancel in the expression of the second variation. Equation (1.2) need not hold if I consider only perturbations which do not increase $r(h)$. Instead, I get the inequality 


$$
\frac{r^{\prime}(h)}{\sqrt{1+\left(r^{\prime}(h)\right)^{2}}}<\beta
$$

(equality is excluded by the preceeding calculation). Physically, what this means is that in the symmetric unbounded liquid bridge problem, the only time a nontrivial stable bridge can be formed is when the entire disc is wetted, and the contact angle between the normal to the surface at the edge of the disc and the downward normal to the disc has cosine less than $\beta$.

Even when a nontrivial symmetric liquid bridge has a constraint on $r(h)$, the profile curve must still satisfy (1.1). I may assume that $\kappa=1$ by making the usual transformation

$$
\frac{1}{\sqrt{\kappa}} r(\sqrt{\kappa} u) \longrightarrow r(u),
$$

so that I have:

$$
\frac{r r^{\prime \prime}}{\left(1+\left(r^{\prime}\right)^{2}\right)^{3 / 2}}-\frac{1}{\sqrt{1+\left(r^{\prime}\right)^{2}}}=u r \text {. }
$$

I no longer have the initial condition (1.2). The profile curves which satisfy (1.7) and the condition that $\lim _{u \rightarrow 0} r(u)=+\infty$ form a one-parameter family (see Figure 2). It is inconvenient, however, to express this parametrization when the curves are considered as functions of $u$. This leads us to the next section.

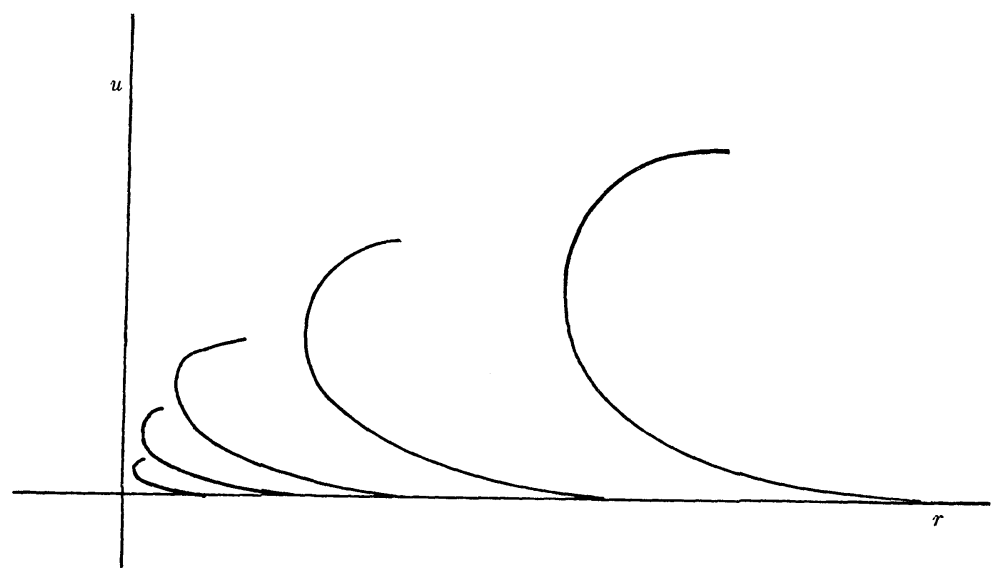

FIGURE 2

2. The profile curves in parametric form. Equation (1.7) can be written in a more elegant and useful form parametrically. Fix a profile curve $r(u)$ and let $\psi(u)$ be the angle $\pi / 2-\tan ^{-1}\left(r^{\prime}(u)\right)$. This 


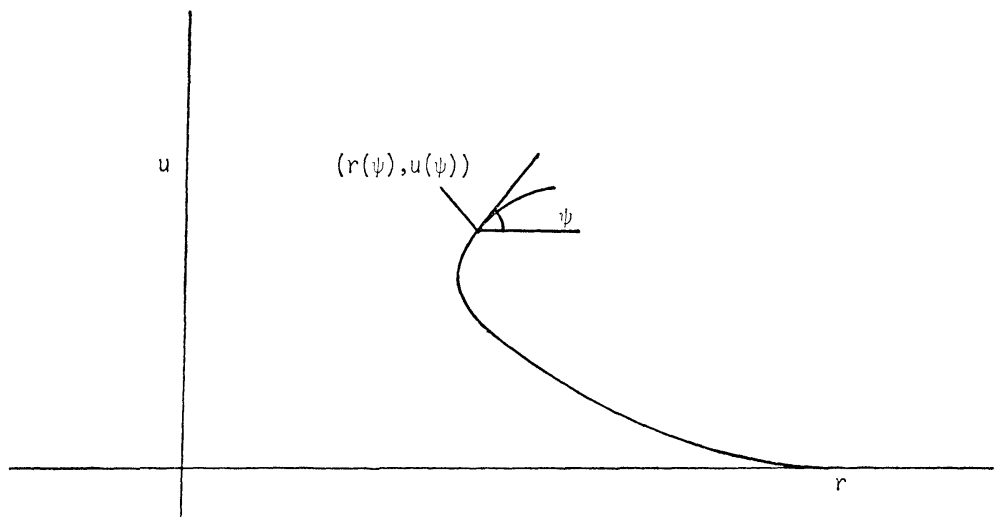

FIGURE 3

Parametrization by the inclination angle $\psi$

is called the inclination angle of the curve (see Figure 3). Clearly $d \psi / d u<0$, so $\psi(u)$ can be inverted to obtain $u(\psi)$. The radius, then, is expressible as $r(\psi)=r(u(\psi))$. The differential equations that $r(\psi)$ and $u(\psi)$ satisfy are:

$$
\frac{d u}{d \psi}=\frac{-r \sin \psi}{r u+\sin \psi},
$$

and

$$
\frac{d r}{d \psi^{\prime}}=\frac{-r \cos \psi}{r u+\sin \psi} .
$$

The method of parametrizing each curve by the inclination angle is used by Finn for the sessile drop [4], where he obtains equations similar to the two above. Wente [15] obtained equations (2.1) and (2.2) for the pendent drop. Finding appropriate initial conditions for these equations in our case is something of a problem. The portions of the profile curves from $\psi=\pi / 2$ to $\psi=\pi$ have already been studied, as they are the solutions to the symmetric exterior capillary problem (see Johnson and Perko [8], Siegel [13], and Turkington [14]). In particular, Johnson and Perko have shown that for a given radius $\sigma>0$, there is a unique curve satisfying (2.1), (2.2), $\lim _{u \rightarrow 0} v(u)=+\infty$, and being vertical at radius $\sigma$. Let $T(\sigma)$ be the height of the vertical point of that curve which is vertical at radius $\sigma$ (see Figure 4). Turkington has shown that $T(\sigma) \sim \sigma \log (1 / \sigma)$ as $\sigma$ approaches zero, and $T(\sigma) \sim \sqrt{2}$ as $\sigma$ approaches $+\infty$. Siegel has shown that $T(\sigma)$ is strictly increasing in $\sigma$. The boundary conditions for $r(\psi ; \sigma)$ and $u(\psi ; \sigma)$ are: 


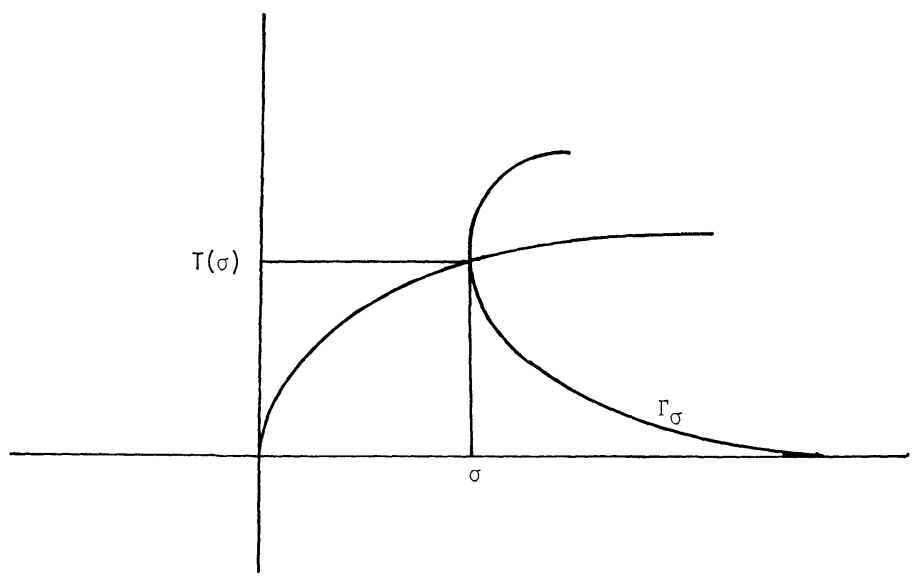

FigURE 4

The Curve of Vertical Points $T^{\prime}(\sigma)$

$$
\begin{gathered}
r\left(\frac{\pi}{2} ; \sigma\right)=\sigma, \\
u\left(\frac{\pi}{2} ; \sigma\right)=T(\sigma) .
\end{gathered}
$$

Later in this paper I will show that $T(\sigma) \in C^{1}(0,+\infty)$.

Siegel has shown that for $\psi \geqq \pi / 2$, it is impossible to have two distinct profile curves having the same inclination angle $\psi$ at the same height $u$. Formally, if $u\left(\psi_{0} ; \sigma_{1}\right)=u\left(\psi_{0} ; \sigma_{2}\right)$ for $\pi>\psi_{0} \geqq \pi / 2$, then $\sigma_{1}=\sigma_{2}$. Theorem 2.3 will extend this to the entire range $\pi>\psi \geqq 0$. I first need the following lemmas:

Lemma 2.1. Let $\Gamma_{\sigma}=(r(\psi ; \sigma), u(\psi ; \sigma))$ be a particular profile curve. Pick $\psi_{0} \in[0, \pi)$, and let $r_{0}=r\left(\psi_{0} ; \sigma\right)$ and $u_{0}=u\left(\psi_{0} ; \sigma\right)$. Then the volume of the solid obtained by rotating the unbounded region with boundary $u=u_{0}, u=0, r=0$, and $\Gamma_{o}$ around the $u$ axis is

$$
2 \pi r_{0} \sin \psi_{0}+\pi r_{0}^{2} u_{0}
$$

Proof. This is an application of Laplace's formula for the volume contained by a symmetric capillary surface (see Laplace [9]), which is obtained by an integration by parts. The one point to check is that the boundary term at infinity from the integration vanishes. This, however, is easy to verify, using Gerhardt's result that the height of the profile curves decreases faster than $e^{-r}$ as $r$ approaches $+\infty$ (see Gerhardt [5]).

This lemma thus gives an easy way to find the volume of liquid 
lifted by a disc if the radius $r_{0}$ of the disc, the height $u_{0}$ of the disc above the base plane, and the contact angle $\psi_{0}$ of the surface with the disc are known. The next lemma is a technical one, used in the proof of Theorem 2.3.

LEMma 2.2. Preserving the notation of Lemma 2.1, let $\psi_{0} \in$ $[0, \pi / 2]$. Let $A$ be the solid obtained by rotating the region bounded by $r=r_{0}$ and $\Gamma_{\sigma}$ and let $B$ be the solid obtained by rotating the unbounded region between $\Gamma_{\sigma}$ and the $r$ axis from $r=r_{0}$ to $r=+\infty$ (see Figure 5). Then

$$
|B|-|A|=2 \pi r_{0} \sin \psi_{0} .
$$

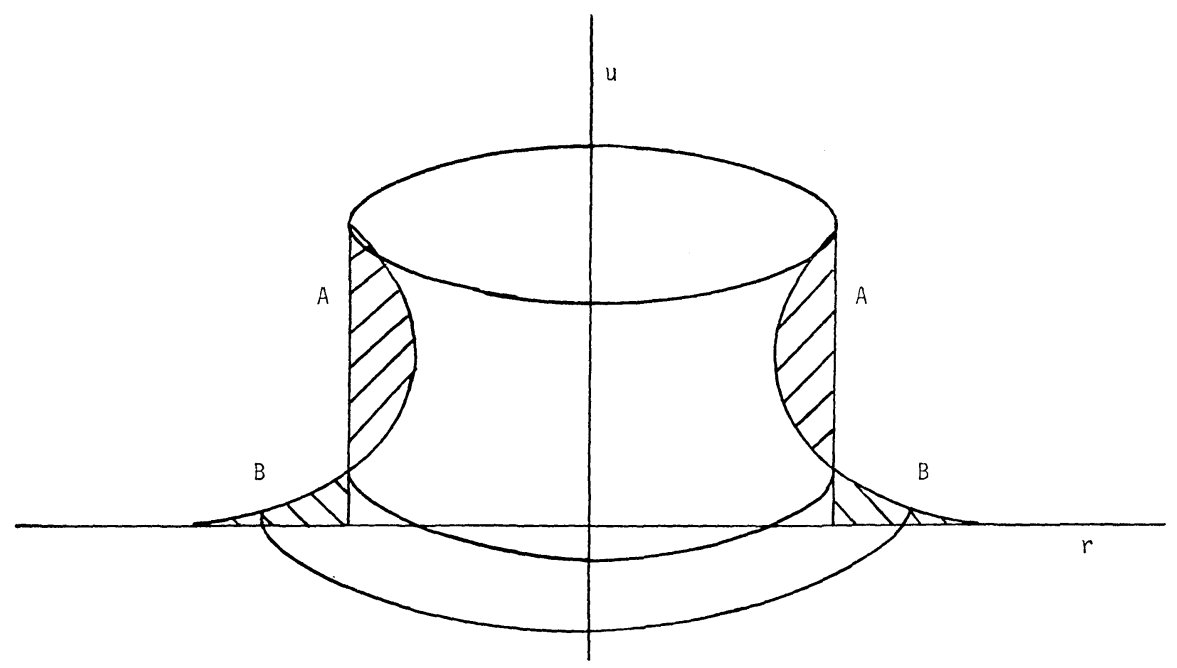

Figure 5

Proof of Lemma 2.2

Proof. The volume of the solid described in Lemma 2.1 is $|B|-|A|+\pi r_{0}^{2} u_{0}$, from which the result follows.

THEOREM 2.3. If $u\left(\psi_{0} ; \sigma_{1}\right)=u\left(\psi_{0} ; \sigma_{2}\right)$ for $\psi_{0} \in[0, \pi)$, then $\sigma_{1}=\sigma_{2}$, that is, the profile curves are the same. (As mentioned above, this has been proven by Siegel for $\left.\psi_{0} \in[\pi / 2, \pi)\right)$.

Proof. I will proceed by contradiction. Let $\Gamma_{1}$ and $\Gamma_{2}$ (corresponding to $\sigma_{1}$ and $\sigma_{2}$ respectively) be two profile curves contradicting the conclusion of the theorem, and suppose $\sigma_{1}<\sigma_{2}$. There is a largest angle $\psi_{0}<\pi / 2$ for which $u\left(\psi_{0} ; \sigma_{1}\right)=u\left(\psi_{0} ; \sigma_{2}\right)$, since we know that this equality cannot hold for $\psi_{0} \geqq \pi / 2$. By the convexity of the profile curves, $u_{0}=u\left(\psi_{0} ; \sigma_{1}\right)=u\left(\psi_{0} ; \sigma_{2}\right)$ is the lowest height at which $\Gamma_{1}$ and $\Gamma_{2}$ have the same inclination angle. For ease of 
notation, let $\Gamma_{1}$ and $\Gamma_{2}$ stand for the parts of the profile curves beneath $u=u_{0}$ for the rest of the proof.

It is clear that $\Gamma_{1}$ and $\Gamma_{2}$ cannot cross more than once, for if they did, there would be a height $u_{1}<u_{0}$ at which $\Gamma_{1}$ and $\Gamma_{2}$ have the same inclination angle. I therefore have two cases to consider: case I, that $\Gamma_{1}$ and $\Gamma_{2}$ do not cross, and case II, $\Gamma_{1}$ and $\Gamma_{2}$ cross precisely once.

Case I. (See Figure 6.) Since $T(\sigma)$ is strictly increasing, there must hold:

$$
u\left(\frac{\pi}{2} ; \sigma_{1}\right)<u\left(\frac{\pi}{2} ; \sigma_{2}\right)
$$

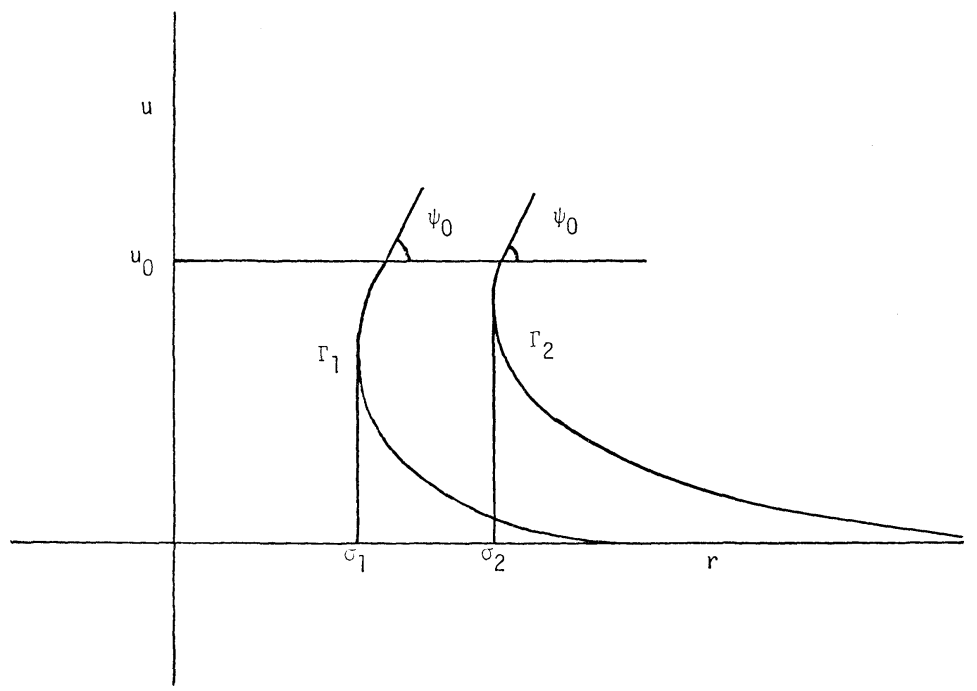

FIgURE 6

Theorem 2.3, Case I

Rewrite equation (2.1) as:

$$
\frac{d u}{d \psi}=\frac{-\sin \psi}{u+\frac{\sin \psi}{r}} .
$$

Because $r\left(\psi_{0} ; \sigma_{1}\right)<r\left(\psi_{0} ; \sigma_{2}\right)$ (the curves do not cross), it follows that:

$$
0>\left.\frac{d u\left(\psi ; \sigma_{1}\right)}{d \psi}\right|_{\psi=\psi_{0}}>\left.\frac{d u\left(\psi ; \sigma_{2}\right)}{d \psi}\right|_{\psi=\psi_{0}}
$$

Thus, $u\left(\psi ; \sigma_{1}\right)>u\left(\psi ; \sigma_{2}\right)$ in some open interval $\left(\psi_{0}, \hat{\psi}\right)$. But from this and (2.5), it follows that there is a $\psi_{1} \in\left(\psi_{0}, \pi / 2\right)$ with $u\left(\psi_{1} ; \sigma_{1}\right)=$ 
$u\left(\psi_{1} ; \sigma_{2}\right)$, contradicting the choice of $\psi_{0}$. This eliminates case I.

Case II. (See Figure 7.) Let $A_{1}$ and $B_{1}$ be the solids described in Lemma 2.2 corresponding to $\Gamma_{1}$, and similarly with $A_{2}$ and $B_{2}$.

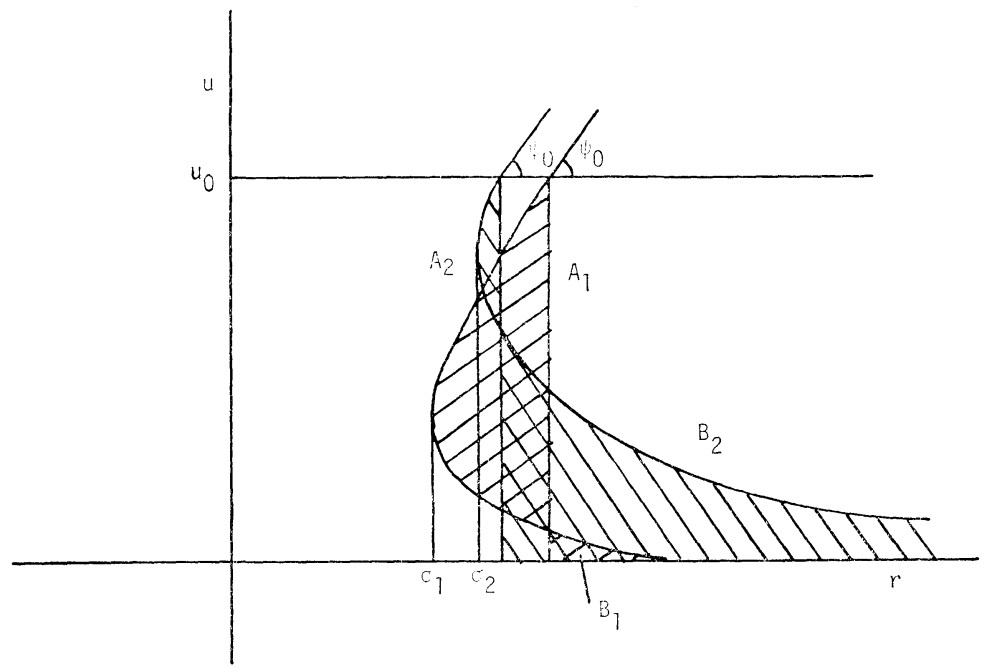

FiguRe 7

Theorem 2.3, Case II

Since $\sigma_{1}<\sigma_{2}$, it follows (see Johnson and Perko) that the height at which $\Gamma_{1}$ recrosses the line $r=r\left(\psi_{0} ; \sigma_{1}\right)$ is less than the height at which $\Gamma_{2}$ crosses $r=r\left(\psi_{0} ; \sigma_{1}\right)$. This implies that $B_{1} \varsubsetneqq B_{2}$, so $\left|B_{1}\right|<$ $\left|B_{2}\right|$.

The next step is to show that $\left|A_{2}\right| \leqq\left|A_{1}\right|$. Since the profile curves cross once, $r\left(\psi_{0} ; \sigma_{1}\right) \geqq r\left(\psi_{0} ; \sigma_{2}\right)$. Let $\Gamma^{\prime}$ be the curve obtained by translating $\Gamma_{2}$ to the right by $r\left(\psi_{0} ; \sigma_{1}\right)-r\left(\psi_{0} ; \sigma_{2}\right)$ units (so that $\Gamma^{\prime}$ is not a member of the family of profile curves). Let $A^{\prime}$ be the solid formed by rotating the bounded region between $\Gamma^{\prime}$ and the line $r=r\left(\psi_{0} ; \sigma_{1}\right)$ around the $u$ axis.

It cannot happen that $\Gamma^{\prime}$ intersects $\Gamma_{1}$ below the height $u_{0}$, for if it did I would have a smaller value of $u$ at which $\Gamma_{1}$ and $\Gamma^{\prime}$ have the same inclination. But then $\Gamma_{1}$ and $\Gamma_{2}$ would have the same inclination at that height, contradicting the choice of $\psi_{0}$. Hence $\Gamma^{\prime}$ lies entirely to the left or entirely to the right of $\Gamma_{1}$.

Since $\sigma_{1}<\sigma_{2}$, we must have $\Gamma_{2}$ to the right of $\Gamma_{1}$ for $\psi>\pi / 2$ (see Johnson and Perko), hence $\Gamma^{\prime}$ lies entirely to the right of $\Gamma_{1}$. I may then conclude that $\left|A^{\prime}\right| \leqq\left|A_{1}\right|$. However, since $r\left(\psi_{0} ; \sigma_{1}\right) \geqq$ $r\left(\psi_{0} ; \sigma_{2}\right)$, I have $\left|A_{2}\right| \leqq\left|A_{1}\right|$. Therefore, I find that

$$
\left|B_{1}\right|-\left|A_{1}\right|<\left|B_{2}\right|-\left|A_{2}\right| \text {. }
$$

This is a contradiction, since 


$$
\left|B_{1}\right|-\left|A_{1}\right|=2 \pi r\left(\psi_{0} ; \sigma_{1}\right) \sin \psi_{0} \geqq 2 \pi r\left(\psi_{0} ; \sigma_{2}\right) \sin \psi_{0}=\left|B_{2}\right|-\left|A_{2}\right|
$$

by Lemma 2.2. This completes the proof of Theorem 2.3.

Corollary 2.4. No two distinct profile curves can cross twice.

As $\sigma$ tends to infinity, the profile curves tend to "look" like the solution to the one-dimensional problem, that is, the surface formed when the disc has expanded to a half-plane. The one-dimensional profile $\left(t_{\infty}(\psi), u_{\infty}(\psi)\right)$ solves the equations:

$$
\begin{aligned}
\frac{d u}{d \psi} & =\frac{-\sin \psi}{u} \\
\frac{d t}{d \psi} & =\frac{-\cos \psi}{u} \\
u_{\infty}\left(\frac{\pi}{2}\right) & =\sqrt{2},
\end{aligned}
$$

and

$$
t_{\infty}\left(\frac{\pi}{2}\right)=0
$$

where the vertical point is on the $u$ axis.

THEOREM 2.5. As $\sigma \rightarrow \infty, r(\psi ; \sigma)-\sigma$ approaches $t_{\infty}(\psi)$ almost uniformly in $\psi \in[0, \pi)$, and $u(\psi ; \sigma)$ approaches $u_{\infty}(\psi)$ almost uniformly in $\psi$.

Proof. Let $t(\psi ; \sigma)=r(\psi ; \sigma)-\sigma$. I can then write:

$$
\begin{gathered}
\frac{d u}{d \psi}=\frac{-\sin \psi}{u+\left(\frac{\sin \psi}{\sigma+t}\right)}, \\
\frac{d t}{d \psi}=\frac{-\cos \psi}{u+\left(\frac{\sin \psi}{\sigma+t}\right)}, \\
u\left(\frac{\pi}{2}\right)=T(\sigma),
\end{gathered}
$$

and

$$
t\left(\frac{\pi}{2}\right)=0
$$

As $\sigma$ goes to infinity, the coefficients of the above equations tend almost uniformly to the coefficients of (2.7). By the theorem 
on continuous dependence of solutions of O.D.E.'s on their parameters, the conclusion of the theorem follows.

REMARK 2.6. It is not difficult to integrate (2.7) to obtain:

$$
\begin{aligned}
& u_{\infty}(\psi)=\sqrt{2 \cos \psi+2} \\
& t_{\infty}(\psi)=\log \left|\sec \left(\frac{\psi}{2}\right)+\tan \left(\frac{\psi}{2}\right)\right|-2 \sin \left(\frac{\psi}{2}\right)+\sqrt{2}-\log (\sqrt{2}+1) .
\end{aligned}
$$

This is the continuation of the one-dimensional solution which was known to Laplace. It has been expressed as a function of height in Bakker [1].

It will be useful to obtain some estimates on the behavior of the profile curves. The first is obtained by a reflection argument.

THEOREM 2.7. $u(\psi ; \sigma)<2 T(\sigma)$ for all $\psi \in[0, \pi)$, and all $\sigma \in$ $(0,+\infty)$.

Proof. Fix $\sigma>0$. The profile curve $\Gamma_{\sigma}$ can be considered as the union of the graphs of two functions of $r, w_{1}(r)$ and $w_{2}(r)$, where $w_{1}$ represents the lower part of the curve and $w_{2}$ the upper part. Let $\psi_{1}(r)$ and $\psi_{2}(r)$ be the corresponding inclination angles. These functions satisfy:

$$
\left(r \sin \psi_{1}\right)_{r}=-r w_{1},
$$

and

$$
\left(r \sin \psi_{2}\right)_{r}=-r w_{2}
$$

with appropriate boundary conditions, since $1 / r(r \sin \psi)_{r}$ is the symmetric mean curvature operator. Define a function $w_{3}(r)$ by:

$$
w_{3}(r)=2 T(\sigma)-w_{1}(r),
$$

so that the graph of $w_{3}(r)$ is the reflection of the graph of $w_{1}$ across the line $u=T(\sigma)$. Let $\psi_{3}$ be the corresponding inclination angle, so that $\psi_{3}(r)=\pi-\psi_{1}(r)$.

I then have:

$$
\left(r \sin \psi_{3}\right)_{r}=-r w_{1}
$$

hence

$$
\left(r \sin \psi_{3}\right)_{r} \geqq\left(r \sin \psi_{2}\right)_{r},
$$

since $w_{2} \geqq w_{1}$.

Moreover, this inequality is strict for $r>\sigma$. I also have 
$\sin \psi_{3}(\sigma)=\sin \psi_{2}(\sigma)$. Integrating (2.10), I get

$$
\sin \psi_{3}(r)>\sin \psi_{2}(r) \quad \text { for } \quad r>\sigma,
$$

so

$$
w_{3}^{\prime}(r)>w_{2}^{\prime}(r) \quad \text { for } \quad r>\sigma .
$$

But $w_{3}(\sigma)=w_{2}(\sigma)=T(\sigma)$, so

$$
w_{3}(r)>w_{2}(r) \quad \text { for } \quad r>\sigma \text {. }
$$

It is clear that $w_{3}(r)<2 T(\sigma)$ for all $r$, from which the desired result follows.

Theorem 2.8. Using the bound in Theorem 2.7, I can estimate $r(0 ; \sigma)$. Integrating (2.9) from $r\left(\psi_{0} ; \sigma\right)$ to $r(0 ; \sigma), \psi_{0} \in[0, \pi / 2]$, I have:

$$
-r\left(\psi_{0} ; \sigma\right) \sin \psi_{0}=\int_{r\left(\psi_{0} ; \sigma\right)}^{r(0 ; \sigma)} d\left(r \sin \psi_{2}\right)=\int_{r\left(\psi_{0} ; \sigma\right)}^{r(0 ; \sigma)}-r w_{2}(r) d r
$$

Putting in the bounds $u\left(\psi_{0} ; \sigma\right) \leqq w_{2}(r) \leqq u(0 ; \sigma)$, there follows:

$$
\begin{aligned}
u\left(\psi_{0} ; \sigma\right)\left(\frac{r^{2}(0 ; \sigma)-r^{2}\left(\psi_{0} ; \sigma\right)}{2}\right) & \leqq r\left(\psi_{0} ; \sigma\right) \sin \psi_{0} \\
& \leqq u(0 ; \sigma)\left(\frac{r^{2}(0 ; \sigma)-r^{2}\left(\psi_{0} ; \sigma\right)}{2}\right)
\end{aligned}
$$

holding for $0 \leqq \psi_{0} \leqq \pi / 2$.

Letting $\psi_{0}=\pi / 2$, the above becomes:

$$
T(\sigma)\left(\frac{r^{2}(0 ; \sigma)-\sigma^{2}}{2}\right) \leqq \sigma \leqq u(0 ; \sigma)\left(\frac{r^{2}(0 ; \sigma)-\sigma^{2}}{2}\right) .
$$

Since $u(0 ; \sigma)<2 T(\sigma)$, I obtain:

$$
T(\sigma)\left(\frac{r^{2}(0 ; \sigma)-\sigma^{2}}{2}\right) \leqq \sigma \leqq T(\sigma)\left(r^{2}(0 ; \sigma)-\sigma^{2}\right) .
$$

It follows that:

$$
\sqrt{\frac{\sigma}{T(\sigma)}+\sigma^{2}} \leqq r(0 ; \sigma) \leqq \sqrt{\frac{2 \sigma}{T(\sigma)}+\sigma^{2}} .
$$

Inequality (2.12) may be used to estimate $r(0 ; \sigma)$ as $\sigma$ goes to zero. Since $T(\sigma) \sim \sigma \log (1 / \sigma)$, and

$$
\sigma \sqrt{1+\frac{1}{\sigma T(\sigma)}} \leqq r(0 ; \sigma) \leqq \sigma \sqrt{1+\frac{2}{\sigma T(\sigma)}},
$$

it follows that: 


$$
r(0 ; \sigma)=O\left(\frac{1}{V\left(\frac{1}{\log (1 / \sigma)}\right.}\right)
$$

as $\sigma \rightarrow 0$.

To get some idea of the behavior of the curve $(r(0 ; \sigma), u(0 ; \sigma))$ as $\sigma$ approaches zero, consider the ratio $u(0 ; \sigma) / \imath(0 ; \sigma)$. From the preceding estimates it follows that:

$$
0<\frac{u(0 ; \sigma)}{\imath^{\circ}(0 ; \sigma)}<\frac{2 T(\sigma)}{\sqrt{\sigma^{2}+\frac{\sigma}{T(\sigma)}}}=\frac{2}{\sqrt{\frac{\sigma^{2}}{T^{2}(\sigma)}+\frac{\sigma}{T^{2}(\sigma)}}} .
$$

But

$$
\lim _{\sigma \rightarrow 0} \frac{\sigma}{T^{3}(\sigma)}=+\infty
$$

thus

$$
\lim _{\sigma \rightarrow 0} \frac{u(0 ; \sigma)}{r(0 ; \sigma)}=0
$$

From this I conclude that the curve of horizontal points $(r(0 ; \sigma), u(0 ; \sigma))$ becomes horizontal at $(0,0)$. This is in contrast to the fact that the curve of vertical points $(\sigma, T(\sigma))$ becomes vertical at the origin.

REMARK 2.9. The fact that $(r(0 ; \sigma), u(0 ; \sigma))$ goes to $(0,0)$ forces the curves $\left(r\left(\gamma_{0} ; \sigma\right), u\left(\psi_{0} ; \sigma\right)\right)$ to go to the origin for $0 \leqq \psi_{0} \leqq \pi / 2$. This, in combination with Theorem 2.3 shows that the curves $\left(r\left(\psi_{0} ; \sigma\right), u\left(\psi_{0} ; \sigma\right)\right)$ are graphs of functions of height defined on the range $\left(0, u_{\infty}\left(\psi_{0}\right)\right)$. (This is already known for $\left.\pi \geqq \psi_{0} \geqq \pi / 2\right)$. To be specific, if $\sigma_{1}>\sigma_{2}$, then $u\left(\psi_{0} ; \sigma_{1}\right)>u\left(\psi_{0} ; \sigma_{2}\right)$.

REMARK 2.10. All of the profile curves must lie beneath the curve $u=2 T(r)$. This comes from Theorem 2.7, the fact that $\sigma \leqq r(\psi ; \sigma)$ for all $\psi$, and the monotonicity of $T(\sigma)$.

3. Differentiability of $T(\sigma)$. I will need to differentiate equations (2.3) and (2.4) with respect to $\sigma$, so it is necessary to show that $T(\sigma) \in C^{1}(0, \infty)$. To do this, first define a slope function $p(R, u)$ on the region $R \in(0, \infty), u \in(0, T(R))$ by $p(R, u)=d u /\left.d v\right|_{r=R}$ for that unique profile curve $\left(r\left(\psi ; \sigma_{0}\right), u\left(\psi ; \sigma_{0}\right)\right)$ with $r\left(\psi_{0} ; \sigma_{0}\right)=R$ and $u\left(\psi_{0} ; \sigma_{0}\right)=u$ for some $\psi_{0} \in(\pi / 2, \pi)$. Of course, $p(R, u)=\tan \psi_{0}$ for this $\psi_{0}$. That this is a valid definition is seen from Lemma 4.1 of Johnson and Perko [8]. In this $\S I$ will only be working with the lower parts of 
the profile curves, $\psi \in(\pi / 2, \pi)$.

The plan of action is to show that for fixed $R$ sufficiently large, $p(R, u)$ is $C^{1}$ in $u$ for $u$ sufficiently small. Once I have that, I will be able to prove some regularity results by the theorem on continuous dependence of solutions of O.D.E.'s on their parameters. I need to know some properties of $p(R, u)$. Johnson and Perko prove the following:

Lemma 3.1. For fixed $R, p(R, u)$ is continuous, negative, decreasing in $u$, with:

$$
\lim _{u \rightarrow 0} p(R, u)=0,
$$

and

$$
\lim _{u \rightarrow T(R)} p(R, u)=-\infty .
$$

I have to be able to refer to the lower parts of the profile curves as functions of $r$, so define $w(r ; R, u)$ to be the portion of that profile curve from $\psi=\pi / 2$ to $\psi=\pi$ which passes through $(R, u)$. (This is only for $0<u \leqq T(R)$.) If I let $w^{\prime}$ be $(\partial / \partial r) w(r ; R, u)$, then $w(r ; R, u)$ satisfies (again from Johnson and Perko):

$$
w^{\prime \prime}=w\left(1+\left(w^{\prime}\right)^{2}\right)^{3 / 2}-\frac{w^{\prime}}{r}\left(1+\left(w^{\prime}\right)^{2}\right),
$$

with boundary conditions:

(a) $w(R ; R, u)=u$

(b) $\lim _{r \rightarrow \infty} w(r ; R, u)=0$

(c) $w^{\prime}(R ; R, u)=p(R, u)$,

where (3.2) (c) is the definition of $p(R, u)$.

I will need to know some properties of $w(r ; R, u)$.

LEMmA 3.2. Let $R>0, T(R)>u>0, r \geqq R, u_{1}<u_{2}$. Then:

(i) $w(r ; R, u)>0, w^{\prime}(r ; R, u)<0, w^{\prime \prime}(r ; R, u)>0$,

(ii) $0<w\left(r ; R, u_{1}\right)<w\left(r ; R, u_{2}\right)$

(iii) $0>w^{\prime}\left(r ; R, u_{1}\right)>w^{\prime}\left(r ; R, u_{2}\right)$

(iv) $0<w^{\prime \prime}\left(r ; R, u_{1}\right)<w^{\prime \prime}\left(r ; R, u_{2}\right)$

(v) $w(r ; R, u)<c e^{-r} / \sqrt{r}, c=c(R, u)$

(vi) $0>w^{\prime}(r ; R, u)>c_{1}(d / d r)\left(e^{-r} / \sqrt{r}\right), c_{1}=c_{1}(R, u)$.

Proof. Part (i) is proved in Johnson and Perko. To see part 
(ii), note that $w\left(R: R, u_{1}\right)<w\left(R ; R, u_{2}\right)$ and if $w\left(r ; R, u_{1}\right)=w\left(r ; R, u_{2}\right)$ for some $r>R$, the curves would be identical. The argument for part (iii) is the same, since if $\mathrm{w}^{\prime}\left(r ; R, u_{1}\right)=w^{\prime}\left(r ; R, u_{2}\right)$ for $r \geqq R$, then by Theorem 2 of Johnson and Perko, the curves would be the same. Part (iv) follows from (ii), (iii), and (3.1). Part (v) is proved by Siegel [13], p. 49, and part (vi) is easily obtained from Siegel's estimates.

LEMmA 3.3. Fix $R_{0}>0, u_{0} \in\left(0, T\left(R_{0}\right)\right)$. Then for sufficiently large $R>R_{0}, p(R, u)$ is differentiable on the interval $\left(0, w\left(R ; R_{0}, u_{0}\right)\right)$.

Proof. Let $u$ and $u+\delta$ be less than $w\left(R ; R_{0}, u\right)$ for $R$ to be chosen later, and $\delta \neq 0$. Let

$$
v_{\hat{o}}(r)=\frac{w(r ; R, u+\delta)-w(r ; R, u)}{\delta} .
$$

Then $v_{\hat{o}}$ satisfies (Siegel [13], p. 57):

$$
v_{o}^{\prime \prime}+v_{\hat{o}}^{\prime}\left(\frac{1}{\gamma^{\prime}}+\frac{q_{\hat{o}}^{\prime}}{q_{\hat{o}}}\right)=\frac{1}{q_{\delta}} v_{\hat{o}},
$$

where

$$
q_{\delta}(r)=\int_{0}^{1}\left\{1+\left[w^{\prime}(r ; R, u)+t\left(w^{\prime}(r ; R, u)-w^{\prime}(r ; R, u+\delta)\right)\right]^{2}\right\}^{-3 / 2} d t
$$

with boundary conditions $v_{\hat{\delta}}(R)=1$ and $\lim _{r \rightarrow \infty} v_{\hat{\delta}}(r)=0$. From Lemma 3.2 , part (iii), I have that $w^{\prime}(r ; R, u)$ and $w^{\prime}(r ; R, u+\delta)$ are bounded by $w^{\prime}\left(r ; R_{0}, u_{0}\right)$. From this it follows that:

$$
q_{i}-1<c\left(u_{0}, R_{0}\right) \frac{e^{-2 r}}{r},
$$

and

$$
q_{o}^{\prime}<c_{1}\left(u_{0}, R_{0}\right) \frac{e^{-2 r}}{r} .
$$

Hence, by a calculation of Siegel, there exists an $R=R\left(R_{0}, u_{0}\right)$ such that for $r>R$, the function $U=A e^{-r} / V \bar{r}$ satisfies:

$$
U^{\prime \prime}+U^{\prime}\left(\frac{1}{r}+\frac{q_{\delta}^{\prime}}{q_{\delta}}\right)>\frac{1}{q_{\delta}} U \text { for } \quad r>R .
$$

I can also choose $R$ large enough so that

$$
\frac{1}{q_{\delta}}>0 \quad r>R,
$$


and

$$
\left(\frac{1}{r}+\frac{q_{\delta}^{\prime}}{q_{\delta}}\right)>0 \quad r>R .
$$

Choose $A$ to be $e^{R} V \bar{R}$, and consider the function $v_{\delta}-U$. From (3.3) and (3.4) it follows that this function cannot have a negative minimum. But $v_{\hat{o}}(R)-U(R)=0$ and $\lim _{r \rightarrow \infty}\left(v_{\hat{o}}(r)-U(r)\right)=0$, so we may conclude that:

$$
v_{\delta}(r) \geqq U(r) \quad \text { for } \quad r>R
$$

independently of $u$ and $\delta$.

The reason for looking at $v_{\delta}(r)$ is that

$$
\begin{aligned}
v_{\hat{o}}^{\prime}(R) & =\frac{w^{\prime}(R ; R, u+\delta)-w^{\prime}(R ; R, u)}{\delta} \\
& =\frac{p(R, u+\delta)-p(R, u)}{\delta},
\end{aligned}
$$

so that if I show that $\lim _{\delta \rightarrow 0} v_{\hat{\delta}}^{\prime}(R)$ exists, I will have shown that $p(R, u)$ is differentiable in $u$. However, from equation (3.5) and the fact that $v_{\hat{o}}(R)=U(R)=1$, it follows that:

$$
v_{\dot{o}}^{\prime}(R) \geqq U^{\prime}(R)>-\infty,
$$

holding independently of $\delta$. From Lemma 3.2, I know that $v_{\dot{o}}^{\prime}(R)$ is bounded above by zero. So, for any sequence $\left\{\delta_{i}\right\}$ going to zero, I can, by taking a subsequence, assume that:

$$
\lim _{i \rightarrow \infty} v_{\delta_{i}}^{\prime}(R)=L \geqq U^{\prime}(R) .
$$

Let $v(r ; u)$ be the function solving

$$
v^{\prime \prime}+v^{\prime}\left(\frac{1}{r}+\frac{q^{\prime}}{q}\right)=\frac{1}{q} v, \quad R_{1}>r \geqq R,
$$

with $v(R ; u)=1, v^{\prime}(R ; u)=L$, and

$$
q(r)=\left(1+\left(w^{\prime}(r ; R, u)\right)^{2}\right)^{-3 / 2},
$$

on some maximal interval of definition of $v,\left[R, R_{1}\right), R_{1} \leqq \infty$. (It should be observed that $v(r ; u)$ also depends on $L$.) From the definition of $q_{\delta}(r)$ and part (iii) of Lemma 3.2, it follows that $q_{\delta}(r)$ converges to $q(r)$ uniformly in $[R,+\infty)$ as $\delta$ tends to zero. By the theorem on continuous dependence of solutions of O.D.E.'s on their parameters, it follows that $v_{\delta_{i}}(r)$ approaches $v(r ; u)$ almost uniformly on the domain of existence of $v(r ; u)$.

I now claim that $R_{1}=+\infty$. On $\left[R, R_{1}\right), v(r ; u)>0$ and 
$v^{\prime}(r ; u)<0$, since $v_{\hat{o}_{i}} \rightarrow v$ almost uniformly. From (3.6) I know that $v^{\prime \prime}(r ; u)>0$. Thus $v(r ; u)$ is monotone decreasing and bounded from below by zero, and $v^{\prime}(r ; u)$ is monotone increasing and bounded from above by zero. Hence $v$ and $v^{\prime}$ are bounded on $\left[R, R_{1}\right)$, and it follows from well known existence theorems that $R_{1}=+\infty$. In addition, the limits $v(\infty ; u)$ and $v^{\prime}(\infty ; u)$ exist and are finite, with $v^{\prime}(\infty ; u) \leqq$ $0 \leqq v(\infty ; u)$. Since $v(r ; u)$ is bounded, $v^{\prime}(\infty ; u)=0$, and since $v^{\prime}(r ; u)$ is bounded, $v^{\prime \prime}(\infty ; u)=0$. From equation (3.6) we then get $v(\infty ; u)=0$. (The preceding argument showing that $R_{1}=\infty$ and $v(\infty ; u)=0$ is similar to one of Johnson and Perko.)

It can be shown, however, that there is only one $v(r ; u)$ satisfying (3.6), $v(R ; u)=1$, and $v(\infty, u)=0$ as follows. Suppose there are two such functions $v_{1}$ and $v_{2}$. Then $v_{1}-v_{2}$ satisfies (3.6), with boundary conditions $\left(v_{1}-v_{2}\right)(R)=0$, and $\left(v_{1}-v_{2}\right)(\infty)=0$. But no function satisfying (3.6) can have a positive maximum or a negative minimum, so $v_{1}-v_{2} \equiv 0$, and $v_{1}(r)=v_{2}(r)$.

Thus, for any sequence of $\delta$ 's going to zero, there is a subsequence $\left\{\delta_{i}\right\}$ with $\lim _{i \rightarrow \infty} v_{s_{i}}^{\prime}(R)=v^{\prime}(R, u)$ where $v^{\prime}(R ; u)$ doesn't depend on the sequence chosen. Hence $\lim _{\delta \rightarrow 0} v_{\delta}^{\prime}(R)$ exists and equals $v^{\prime}(R, u)$, so $p(R, u)$ is differentiable for $0<u<w\left(R ; R_{0}, u_{0}\right)$.

LEMmA 3.4. Under the same assumptions as above, $p(R, u)$ is $C^{1}$ on $\left(0, w\left(R ; R_{0}, u_{0}\right)\right)$ for fixed $R$.

Proof. From the proof of Lemma 3.3, I know that $\partial p / \partial u(R, u)=$ $v^{\prime}(R ; u)$. Pick some $\zeta \in\left(0, w\left(R ; R_{0}, u_{0}\right)\right)$, and let $\left\{\zeta_{i}\right\}$ approach $\zeta$. To show that $\partial p / \partial u(R, u)$ is continuous at $(R, \zeta)$, I must show that $\lim _{i \rightarrow \infty} v^{\prime}\left(R ; \zeta_{i}\right)=v^{\prime}(R ; \zeta)$. As in Lemma 3.3 , by taking a subsequence I can assume that:

$$
\lim _{i \rightarrow \infty} v^{\prime}\left(R ; \zeta_{i}\right)=M>-\infty,
$$

since $e^{R} V \bar{R}\left(e^{-r} / V \bar{r}\right)$ is a subsolution to (3.6). But the coefficients of the equations for $v\left(r ; \zeta_{i}\right)$ are converging to the coefficients of the equation for $v(r ; \zeta)$. Let $v_{1}(r)$ solve (3.6) with $v_{1}^{\prime}(R)=M$. As in the proof of Lemma 3.3, $v_{1}(r)$ is defined on $[R, \infty)$ and is zero at infinity, so $v_{1}(r)=v(r, \zeta)$. Hence $M=v^{\prime}(R ; \zeta)$, so it follows that

$$
\lim _{i \rightarrow \infty} v^{\prime}\left(R ; \zeta_{i}\right)=v^{\prime}(R ; \zeta) \text {. }
$$

Therefore $p(R, u)$ is $C^{1}$ as a function of $u$ on $\left(0, w\left(R ; R_{0}, u_{0}\right)\right)$.

THEOREM 3.5. The slope function $p(r, u)$ is $C^{1}$ in the region $W=\{(r, u) ; 0<r<\infty, 0<u<T(r)\}$. 
Proof. Pick $\left(r_{0}, u_{0}\right) \in W$, and consider the profile curve $w\left(r ; r_{0}, u_{0}\right)$. Define the region $W_{\left(r_{0}, u_{0}\right)}^{+}$to be the set $\{(r, u) \in W ; u \geqq$ $\left.w\left(r ; r_{0}, u_{0}\right)\right\}$, and let $W_{\left(r_{0}, u_{0}\right)}^{-}=W-W_{\left(r_{0}, u_{0}\right)}^{r_{0}}$. From Lemma 3.4, there is an $R \geqq r_{0}$ with $p(R, u) \in C^{1}\left(0, w\left(R ; r_{0}, u_{0}\right)\right)$ as a function of $u$. From Lemma 3.2 and the continuity properties of the profile curves proven by Johnson and Perko, I know that $W_{\left(r_{0}, u_{0}\right)}$ is swept out by the set of curves $\left\{w(r ; R, \xi) ; 0<\xi<w\left(R ; r_{0}, u_{0}\right)\right\}$. I can consider this set as the set of functions satisfying (3.1) with initial conditions $w(R ; R, \xi)=\xi$ and $w^{\prime}(R ; R, \xi)=p(R, \xi)$. These initial conditions are $C^{1}$ in $\xi$, so by the theorem on continuous dependence of solutions of O.D.E.'s on their parameters (see e.g., Petrovsky [10]), it follows that $w(r ; R, \xi)$ and $w^{\prime}(r ; R, \xi)$ are $C^{1}$ functions of $\xi$ and $r$.

To prove that $p(r, u)$ is $C^{1}$ is $W_{\left(r_{0}, u_{0}\right)}^{-}$I must show that given $(r, u) \in W_{\left(r_{0}, u_{0}\right)}$, I can define (at least locally) $\xi(r, u)$ so that $u=w(r ; R, \xi(r, u))$, and $\xi$ is a $C^{1}$ function of $r$ and $u$ (the dependence of $\xi$ on $R$ will be understood). It will then follow that $p(r, u)=$ $w^{\prime}(r ; R, \xi(r, u))$, which is clearly $C^{1}$ in $r$ and $u$.

To define $\xi(r, u)$ implicitly as the solution of

$$
u=w(r ; R, \xi),
$$

I need $(\partial / \partial \xi) w(r ; R, \xi) \neq 0$ to use the implicit function theorem. To simplify notation, let $\dot{w}(r ; R, \xi)$ be $(\partial / \partial \xi) w(r: R, \xi)$. Differentiating (3.1) with respect to the parameter $\xi$, I obtain:

$$
\dot{w}^{\prime \prime}=\left(1+\left(w^{\prime}\right)^{2}\right)^{1 / 2}\left(\dot{w}+\dot{w}\left(w^{\prime}\right)^{2}+3 w w^{\prime} \dot{w}^{\prime}\right)-\frac{\dot{w}^{\prime}}{r}\left(1+3\left(w^{\prime}\right)^{2}\right)
$$

with initial conditions:

$$
\dot{v}(R ; R, \xi)=1,
$$

and

$$
\dot{w}^{\prime}(R ; R, \xi)=\frac{\partial p}{\partial \xi}(R, \xi) .
$$

Since $\partial p / \partial \xi(R, \xi)=v^{\prime}(R ; \xi)$, where $v$ is the auxiliary function from Lemma 3.3, it is not difficult to check that $\partial p / \partial \xi(R, \xi)<0$ if $v$ is to go to zero at infinity. I shall use this fact to show that $\dot{w}^{\prime}<0$ on the interval $(r, R)$, from which it follows that $\dot{w}(r ; R, \xi)>1$, enabling me to solve (3.7) for $\xi$ by the implicit function theorem.

Since $\dot{w}^{\prime}(R ; R, \xi)<0$, let $y$ be the largest number less than $R$ for which $\dot{w}^{\prime}(y ; R, \xi)=0$. Integrating from $y$ to $R$, I have that $\dot{w}(y ; R, \xi)>0$, so from equation (3.8), it follows that $\dot{w}^{\prime \prime}(y ; R, \xi)>0$. But this contradicts the assumption that $\dot{w}^{\prime}(\eta ; R, \xi)<0$ for $\eta \in(y, R)$. Thus there is no such $y$, and $\dot{w}^{\prime}$ is negative on $(r, R)$, which, from 
the discussion above, enables me to solve (3.7) for $\xi$ as a $C^{1}$ function of $r$ and $u$. It follows that $p(r, u)$ is $C^{1}$ in $r$ and $u$ in $W_{\left(r_{0}, u_{0}\right)}$. There was nothing special about the point $\left(r_{0}, u_{0}\right)$; indeed, $r_{0}$ could be as large, and $u_{0}$, as close to $T\left(r_{0}\right)$ as desired. Hence $p(r, u)$ is $C^{1}$ in the region $W$.

LEMma 3.6. The curve $p(r, u)=c$ is the graph of a $C^{1}$ function of $r$ for $-\infty<c<0$.

Proof. From Johnson and Perko I know that the curve $p(r, u)=c$ is the graph of a function of $r$. From the proof of Theorem 3.5, $-\infty<\partial p / \partial u<0$ for all $(r, u) \in W$, so by the implicit function theorem, $p(r, u)=c$ can be solved for $u$ as a $C^{1}$ function of $r$. Call this function $T_{c}(r)$.

Lemma 3.7. For any $r_{1}>0, T_{c}^{\prime}(r)$ is bounded on $\left(r_{1},+\infty\right) u n i$ formly in $r$ and $c$.

Proof. Let $x(r ; R, c)$ denote the unique profile curve with derivative $c$ at $r=R$, so that $x(r ; R, p(r, u))=w(r ; R, u)$. From Siegel [13], p. 42, I have:

$$
x\left(r ; R_{2}, c\right)<\frac{R_{2}}{R_{1}} x\left(\frac{R_{1}}{R_{2}} r ; R_{1}, c\right)
$$

for $R_{1}<R_{2}<r$. Let $r$ approach $R_{2}$ to obtain:

$$
x\left(R_{2} ; R_{2}, c\right) \leqq \frac{R_{2}}{R_{1}} x\left(R_{1} ; R_{1}, c\right) .
$$

But $x(r ; r, c)=T_{c}(r)$, so

$$
T_{c}\left(R_{2}\right)<\frac{R_{2}}{R_{1}} T_{c}\left(R_{1}\right)
$$

It follows that:

$$
\frac{T_{c}\left(R_{2}\right)-T_{c}\left(R_{1}\right)}{R_{2}-R_{1}}<\frac{T_{c}\left(R_{1}\right)}{R_{1}}
$$

Since $\left(r, T_{c}(r)\right) \in W$, I have that $T_{c}(r)<\sqrt{2}$, thus

$$
\frac{T_{c}\left(R_{2}\right)-T_{c}\left(R_{1}\right)}{R_{2}-R_{1}}<\frac{\sqrt{2}}{r_{1}}
$$

in $\left(r_{1},+\infty\right)$, bounding the derivative.

Theorem 3.8. The function $T(\sigma)$ is in $C^{1}(0,+\infty)$. 
Proof. Consider the initial value problem:

$$
\begin{aligned}
& \frac{d u}{d \psi}=\frac{-r \sin \psi}{r u+\sin \psi} \\
& \frac{d r}{d \psi}=\frac{-r \cos \psi}{r u+\sin \psi} \\
& r\left(\frac{3 \pi}{4} ; t\right)=t \\
& u\left(\frac{3 \pi}{4} ; t\right)=T_{-1}(t),
\end{aligned}
$$

which is analogous to equations (2.1) to (2.4).

Since $T_{-1}(t)$ is $C^{1}$ in $t$, it follows that $u(\psi ; t)$ and $r(\psi ; t)$ are $C^{1}$ in $\psi$ and $t$. Differentiating the above equations with respect to the parameter $t$, there holds:

$$
\frac{d \dot{u}}{d \psi}=\sin \psi\left[\frac{\dot{u} r^{2}-\dot{r} \sin \psi}{(r u+\sin \psi)^{2}}\right]
$$

and

$$
\frac{d \dot{r}}{d \psi}=\cos \psi\left[\frac{\dot{u} r^{2}-\dot{r} \sin \psi}{(r u+\sin \psi)^{2}}\right]
$$

with $\dot{r}(3 \pi / 4 ; t)=1, \quad \dot{u}(3 \pi / 4 ; t)=T_{-1}^{\prime}(t), \quad$ where $\quad \dot{r}=\partial r / \partial t(\psi ; t) \quad$ and $\dot{u}=\partial u / \partial t(\psi ; t)$.

The curve $(r(\pi / 2 ; t), u(\pi / 2 ; t))$ is the same as the graph of $T(\sigma)$. The only way that it could fail to be a $C^{1}$ curve is if $\dot{r}\left(\pi / 2 ; t_{0}\right)=$ $\dot{u}\left(\pi / 2 ; t_{0}\right)=0$ for some $t_{0}$. But for that $t_{0}$ there would hold $d \dot{u} / d \psi\left(\pi / 2 ; t_{0}\right)=d \dot{r} / d \dot{\psi}\left(\pi / 2 ; t_{0}\right)=0$, from (3.9) and (3.10), so by the usual uniqueness theorems for ordinary differential equations I would have $\dot{u}\left(\psi ; t_{0}\right)=\dot{r}\left(\psi ; t_{0}\right) \equiv 0$. This contradicts the initial conditions, thus $(r(\pi / 2 ; t), u(\pi / 2 ; t))$ is a $C^{1}$ curve, and so is the graph of $T(\sigma)$.

However, this does not imply immediately that $T(\sigma)$ is a $C^{1}$ function of $\sigma . T^{\prime}(\sigma)$ is continuous where it is finite, but it might go off to infinity. I next show that this cannot occur except at $\sigma=0$. From Johnson and Perko, I know that $T_{c}(\sigma)$ increases to $T(\sigma)$ as $c$ tends to $-\infty$. But $T_{c}^{\prime}(\sigma)$ is uniformly bounded on $\left(r_{1},+\infty\right)$ by $\sqrt{2} / r_{1}$ for all $r_{1}>0$, so it follows that $T^{\prime}(\sigma)$ is also bounded by $\sqrt{2} / r_{1}$ on $\left(r_{1},+\infty\right)$. Hence the only point at which $T^{\prime}(\sigma)$ can blow up is $\sigma=0$. From Turkington's estimates it is seen that $T^{\prime}(\sigma)$ does in fact blow up there.

4. The envelope. The envelope is determined by the condition that: 


$$
\left|\begin{array}{ll}
u^{\prime}(\psi ; \sigma) & r^{\prime}(\psi ; \sigma) \\
\dot{u}(\psi ; \sigma) & \dot{r}(\psi ; \sigma)
\end{array}\right|=0
$$

or equivalently

$$
F(\dot{\psi}, \sigma) \equiv \dot{u}(\psi ; \sigma) \cos \psi-\dot{r}(\psi ; \sigma) \sin \psi=0
$$

(see Finn [4] for this equation), where $\dot{u}(\psi ; \sigma)=\partial u / \partial \sigma(\psi ; \sigma)$ and $\dot{r}(\psi ; \sigma)=\partial r / \partial \sigma(\psi ; \sigma)$. I will show that for any $\sigma$, there is precisely one angle $\psi \in(0, \pi)$ which will solve $F(\psi, \sigma)=0$. I will also show that if $\psi=0$ is another solution to (4.1), then this point will be isolated from the rest of the envelope curve.

Differentiating (2.1) through (2.4) with respect to the parameter $\sigma$, permissible since $T(\sigma) \in C^{1}$, I obtain the following needed equations:

$$
\begin{gathered}
\frac{d \dot{u}}{d \psi}=\sin \psi\left[\frac{\dot{u} r^{2}-\dot{r} \sin \psi}{(r u+\sin \psi)^{2}}\right], \\
\frac{d \dot{r}}{d \dot{\psi}}=\cos \psi\left[\frac{\dot{u} r^{2}-\dot{r} \sin \psi}{(r u+\sin \psi)^{2}}\right], \\
\dot{u}\left(\frac{\pi}{2} ; \sigma\right)=T^{\prime}(\sigma), \\
\dot{r}\left(\frac{\pi}{2} ; \sigma\right)=1 .
\end{gathered}
$$

LEMMA 4.1. If for a fixed $\sigma, \dot{r}\left(\psi_{0} ; \sigma\right)>0$, then $\dot{r}(\psi ; \sigma) \geqq 0$ for $\psi_{0} \leqq \psi<\pi$

Proof. From Remark 2.9 it follows that for fixed $\psi, u(\psi ; \sigma)$ is increasing in $\sigma$, thus $\dot{u}(\psi ; \sigma) \geqq 0$ for all $\psi, \sigma$. This fact and equation (4.3) imply the desired result, since if $\dot{r}(\psi ; \sigma)<0$, we have $d \dot{r} / d \dot{\psi}>0$.

LEMMA 4.2. If $\dot{u}\left(0 ; \sigma_{0}\right)=0$, then $\dot{r}\left(0 ; \sigma_{0}\right)<0$.

Proof. The proof is by contradiction, so there are two cases to eliminate:

Case 1. $\quad \dot{u}\left(0 ; \sigma_{0}\right)=0, \dot{r}\left(0 ; \sigma_{0}\right)=0$.

If this occured, then equations (4.2) and (4.3) would be zero at $\psi=0$. By well-known uniqueness theorems for solutions of O.D.E.'s, the only solution to (4.2) and (4.3) would be $\dot{u}\left(\psi ; \sigma_{0}\right)=\dot{r}\left(\psi ; \sigma_{0}\right) \equiv 0$. 
This violates (4.4) and (4.5).

Case 2. $\dot{u}(0 ; \sigma)=0, \dot{r}(0 ; \sigma)>0$.

From (4.2) I have $d \dot{u} /\left.d \psi\right|_{\psi=0}=0$. By L'Hôpital's rule, it follows that:

$$
\lim _{\psi \rightarrow 0} \frac{\dot{u}(\psi ; \sigma)}{\psi}=0
$$

Hence

$$
\lim _{\psi \rightarrow 0}\left(\frac{\dot{u} r^{2}-\dot{r} \sin \psi}{\psi}\right)<0,
$$

so for small positive $\psi, \dot{u} r^{2}-\dot{r} \sin \psi<0$. From (4.2), it follows that $d \dot{u} / d \psi<0$ in $(0, \eta)$ for some positive $\eta$. But this contradicts Lemma 4.1, eliminating this case also.

LeMma $4.3 . \dot{u}(\psi ; \sigma)>0$ for $\psi \in(0, \pi)$.

Proof. Suppose $\dot{u}\left(\psi_{0} ; \sigma\right)=0$ for $\psi_{0} \in(0, \pi)$. There must hold $d \dot{u} /\left.d \psi\right|_{\psi=\psi_{0}}=0$ from the nonnegativity of $\dot{u}$. From this fact and equations (4.2) and (4.3), it follows that $\dot{r}\left(\psi_{0} ; \sigma\right)=0$ and $d \dot{r} /\left.d \psi\right|_{\psi=\psi_{0}}=0$. But this gives as the unique solution $\dot{u}(\psi ; \sigma)=\dot{r}(\psi ; \sigma) \equiv 0$, a contradiction.

THEOREM 4.4. On any profile curve $\Gamma_{o}$ there is exactly one angle $\hat{\psi}(\sigma) \in(0, \pi)$ for which $F(\hat{\psi}(\sigma), \sigma)=0$. In addition, $\hat{\psi}(\sigma)<\pi / 2$.

Proof. $F(\psi, \sigma)>0$ for $\psi$ near zero by Lemmas 4.2 and 4.3 , and $F(\pi / 2, \sigma)=-1$, so there is at least one intermediate zero. I have that $F(\psi, \sigma)<0$ for $\psi>\pi / 2$, since $\dot{r}(\psi ; \sigma) \geqq 0$ for $\psi>\pi / 2$ by Lemma 4.1. I need concern myself, then, only with $\psi \in(0, \pi / 2)$. Let $\psi_{1}$ be the first zero of $F$ on $\Gamma_{\sigma}$. From (4.1) and Lemma 4.3, it follows that $\dot{r}\left(\psi_{1} ; \sigma\right)>0$, and therefore $\dot{r}(\psi ; \sigma) \geqq 0$ for $\psi>\psi_{1}$ from Lemma 4.1. However,

$$
\frac{\partial F}{\partial \psi}=-\dot{u}(\psi ; \sigma) \sin \psi-\dot{r}(\psi ; \sigma) \cos \psi,
$$

so for $\psi>\psi_{1}, \partial F / \partial \psi<0$, and $F$ cannot have a second zero.

It is important to note that I haven't ruled out the possibility that $F(0, \sigma)=0$ for some $\sigma$. However, this is the same as $\dot{u}(0 ; \sigma)=0$, and since $u(0 ; \sigma)$ is increasing in $\sigma$, this can happen only for isolated $\sigma$. These isolated points, then, could not be part of the envelope 
curve given by $\hat{\psi}(\sigma)$ which can be shown to be continuous in $\sigma$. From now on, I will exclude the exceptional case $\psi=0$ from the discussion of the envelope.

I should point out that Theorem 4.4 does not imply that a profile curve cannot intersect the envelope twice in $(r, u)$ space.

Let $E(\sigma): \boldsymbol{R} \rightarrow \boldsymbol{R}^{2}$ be given by $E(\sigma)=(r(\hat{\psi}(\sigma) ; \sigma), u(\hat{\psi}(\sigma) ; \sigma))$, so that this is a parametrization of the envelope.

TheOREM 4.5. As $\sigma$ approaches $+\infty, u(\hat{\psi}(\sigma) ; \sigma)$ approaches $u_{\infty}(0)=2$, so that $E(\sigma)$ has the line $u=2$ as an asymptote.

Proof. As $\sigma$ goes to infinity, $r$ does also. Equations (4.2) and (4.3) therefore approach

$$
\begin{aligned}
& \frac{d \dot{u}}{d \psi}=\sin \psi\left(\frac{\dot{u}}{u_{\infty}^{2}(\psi)}\right), \\
& \frac{d \dot{r}}{d \dot{\psi}}=\cos \psi\left(\frac{\dot{u}}{u_{\infty}^{2}(\psi)}\right),
\end{aligned}
$$

uniformly, with initial conditions

$$
\begin{aligned}
& \dot{u}\left(\frac{\pi}{2}\right)=0, \\
& \dot{r}\left(\frac{\pi}{2}\right)=1 .
\end{aligned}
$$

This has the unique solution $\dot{u} \equiv 0, \dot{r} \equiv 1$. So, by the theorem on continuous dependence of solutions of O.D.E.'s on their parameters, $\dot{u}(\psi ; \sigma)$ goes to zero uniformly in $\psi$ as $\sigma$ goes to infinity, and $\dot{r}(\psi ; \sigma)$ similarly approaches 1 . Hence as $\sigma$ goes to infinity, $\widehat{\psi}(\sigma)$ approaches zero, yielding the desired conclusions.

REMARK 4.6. $E(\sigma)$ lies beneath the curve $u=2 T(r)$ (from Remark 2.10) and therefore goes to zero at $r=0$.

I was unable to advance much further in my study of the envelope without making the following assumption:

Conjecture 4.7. The envelope is a $C^{1}$ curve.

I did not find a way to prove this. I expect it to be true, since the analogous result has been proven for the pendent drop (see Wente [15]). It is well-known that if the envelope of a family of differentiable curves is differentiable, then at each point of this envelope there is a unique member of the family tangent to it. From this, and the fact that $0<\hat{\psi}(\sigma)<\pi / 2$, we may conclude from 4.7 that 
$E(\sigma)$ is the graph of an increasing function of $r$.

THEOREM 4.8. Assuming 4.7, it follows that no profile curve may cross the envelope.

Proof. Case 1. Suppose that for some $\sigma_{0}, \Gamma_{0}$ (corresponding to $\sigma_{0}$ ) crosses $E(\sigma)$ twice at heights $u_{1}$ and $u_{2}$. There then would be a height $u_{0}$ between $u_{1}$ and $u_{2}$ at which $E(\sigma)$ and $\Gamma_{0}$ have the same inclination. But then there would be a $\sigma_{1} \neq \sigma_{0}$ with $u\left(\hat{\psi}\left(\sigma_{1}\right) ; \sigma_{1}\right)=u_{0}$, so, since $E(\sigma)$ is assumed to be $C^{1}, \Gamma_{0}$ and $\Gamma_{1}$ would have the same inclination angle at height $u_{0}$, contradicting Theorem 2.3.

Case 2. Suppose that $\Gamma_{0}$ crosses $E(\sigma)$ once. Then the point of crossing must of course be $\left(r\left(\hat{\psi}\left(\sigma_{0}\right) ; \sigma_{0}\right), u\left(\hat{\psi}\left(\sigma_{0}\right) ; \sigma_{0}\right)\right)$, and $E(\sigma)$ has inclination $\hat{\psi}\left(\sigma_{0}\right)$ there (otherwise I can use the argument of Case 1 here). Since $\Gamma_{0}$ crosses $E$ only once, we cannot have $\Gamma_{0}$ above $E$ at height less than $u\left(\hat{\psi}\left(\sigma_{0}\right) ; \sigma_{0}\right)$. Thus the only possibility to consider is that $\Gamma_{0}$ lies below $E$ for angles greater than $\hat{\psi}\left(\sigma_{0}\right)$ and above $E$ for angles less than $\hat{\psi}\left(\sigma_{0}\right)$ (see Figure 8).

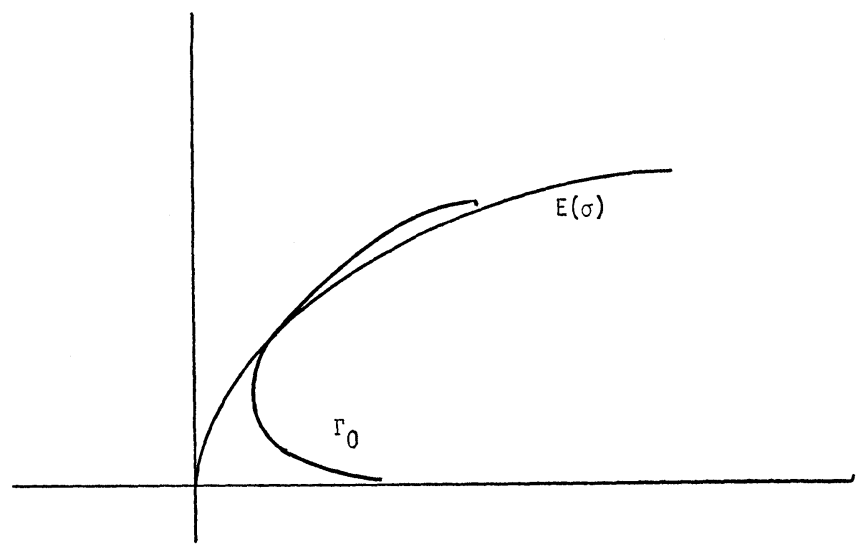

Figure 8

Proof of Theorem 4.8

From Theorem 4.5, $E(\sigma)$ has the line $u=2$ as an asymptote. Since $u(0 ; \sigma)$ increases also to 2 as $\sigma$ approaches infinity, it follows that $u\left(0 ; \sigma_{0}\right)<2$. Since $\hat{\psi}(\sigma)>0$ for all $\sigma$, the envelope is never horizontal, hence at height $v\left(0 ; \sigma_{0}\right), E$ has a steeper inclination than $\Gamma_{0}$. However, for $u$ slightly larger than $u\left(\hat{\psi}\left(\sigma_{0}\right) ; \sigma_{0}\right), \Gamma_{0}$ is steeper than $E$, since $\Gamma_{0}$ rises above $E$. From this I conclude that there is a height between $u\left(0 ; \sigma_{0}\right)$ and $u\left(\hat{\psi}\left(\sigma_{0}\right) ; \sigma_{0}\right)$ at which $E$ and $\Gamma_{0}$ have the same inclination angle. This leads to a contradiction by the same argument as in Case 1. 
THEOREM 4.9. Assuming 4.7, it follows that through any point $\left(r_{0}, u_{0}\right)$ there pass at most two profile curves $\Gamma_{1}$ and $\Gamma_{2}$. In addition, if $u\left(\hat{\psi}\left(\sigma_{1}\right) ; \sigma_{1}\right)<u_{0}$, then $u\left(\hat{\psi}\left(\sigma_{2}\right) ; \sigma_{2}\right)>u_{0} . \quad$ (If $\left(r_{0}, u_{0}\right)$ is between the envelope and the $r$ axis, there will be at least one profile curve through it.)

Proof. From Theorem 4.8, I may assume that $\left(r_{0}, u_{0}\right)$ is not above the envelope. I may also assume that $\left(r_{0}, u_{0}\right) \notin E(\sigma)$, since there will be only one profile curve intersecting any given point of $E(\sigma)$. The theorem will follow if it is shown that there is at most one profile curve through $\left(r_{0}, u_{0}\right)$ touching $E(\sigma)$ at a height below $u_{0}$, and at most one profile touching above $u_{0}$.

To prove the first part of this statement, suppose that there are two profile curves $\Gamma_{1}$ and $\Gamma_{1}^{\prime}$ passing through $\left(r_{0}, u_{0}\right)$ and contacting the envelope at a height lower than $u_{0}$. By Theorem 4.8, $\Gamma_{1}$ and $\Gamma_{1}^{\prime}$ cannot cross $E(\sigma)$. From this and the fact that the profiles are functions of height defined down to $u=0$, it is easy to see that $\Gamma_{1}$ and $\Gamma_{1}^{\prime}$ must cross again, violating Corollary 2.4. Thus there is at most one profile through $\left(r_{0}, u_{0}\right)$ touching the envelope below $u_{0}$.

The proof of the second part is similar. Suppose that $\Gamma_{2}$ and $\Gamma_{2}^{\prime}$ pass through $\left(r_{0}, u_{0}\right)$ and that $u\left(\hat{\psi}\left(\sigma_{2}\right) ; \sigma_{2}\right)>u\left(\hat{\psi}\left(\sigma_{2}^{\prime}\right) ; \sigma_{2}^{\prime}\right)>u_{0} . \quad \Gamma_{2}$ and $\Gamma_{2}^{\prime}$ can't cross again, but what could happen in this case is that $\Gamma_{2}$ could go beyond the horizontal point of $\Gamma_{2}^{\prime}$ and reach the envelope in that way (see Figure 9). In this case, however, $\Gamma_{2}$ has a larger inclination angle than $\Gamma_{2}^{\prime}$ at height $u\left(0 ; \sigma_{2}^{\prime}\right)$, but at $u_{0}, \Gamma_{2}$ has a smaller inclination. This leads to the usual contradiction with

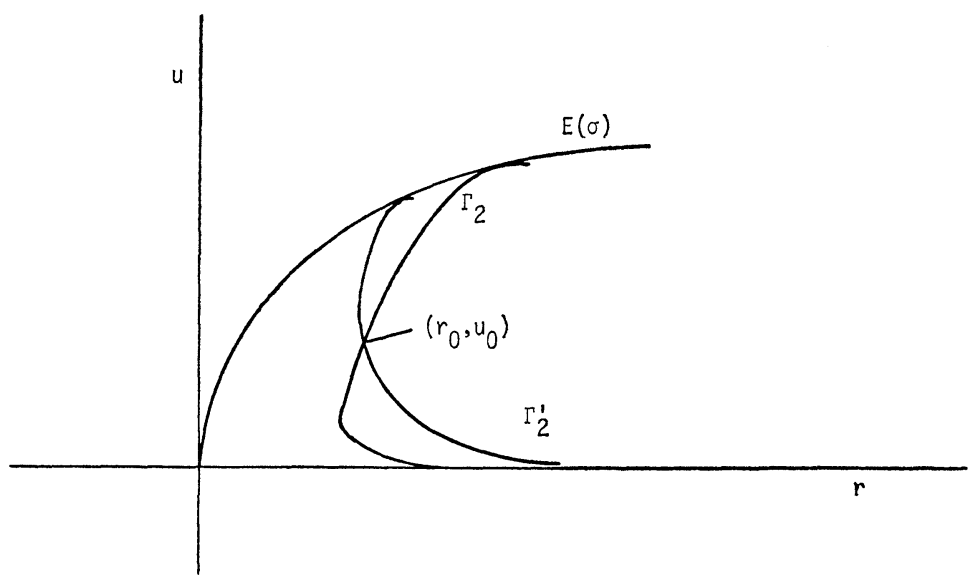

FIGURE 9

Proof of Theorem 4.9 
Theorem 2.3.

5. Stability. In this section I will always be assuming Conjecture 4.7. I will obtain the following results: In the completely wetting case $\beta=1$ (see equation (0.1)), the profile curves are energy minimizing over compact perturbations below the point of contact with the envelope, but once a profile has touched the envelope, it is unstable. In the case $\beta<1$, the curve is unstable if it has touched the envelope or has inclination angle less than $\cos ^{-1} \beta$, otherwise it is stable. A good reference for the methods from the calculus of variations needed in this section, especially the concept of a field of extremals, is Bliss [2].

The first problem to eliminate is the fact that I do not know a priori that if a bridge is stable under compact symmetric perturbations then it is stable under compact asymmetric perturbations as well. To handle this difficulty, I first need the following technical lemma:

LEMmA 5.1. Let $A$ be a set containing $\boldsymbol{R}^{n-1} \times \boldsymbol{R}^{-}$, with the property that

$$
a(\delta)=\inf _{x \in \mathbf{R}^{n-1}}\{|x| \mid(x, \delta) \in \partial A\}
$$

goes to infinity as $\delta$ goes to zero ( $I$ have the liberty to redefine $A$ on a set of measure zero for this). Let $B$ containing $\boldsymbol{R}^{n-1} \times \boldsymbol{R}^{-}$be a compact perturbation of $A$, with $A \Delta B \subseteq K$, where $A \Delta B=(A-B) \cup$ $(B-A)$. Given $\varepsilon>0$, there is a $\delta>0$ and a set $\hat{B}$ with $A \Delta \widehat{B} \subseteq$ $K \cap\{u \geqq \delta\}$, and $\mathscr{E}_{K}(\hat{B})<\mathscr{E}_{K}(B)+\varepsilon$.

Proof. Let $B^{\delta}=(B \cap\{u \geqq \delta\}) \cup(A \cap\{u \leqq \delta\})$ for any $\delta>0$. Since $K$ is compact, by the condition on $\partial A$ it follows that $(\partial A \cap\{u<\delta\}) \cap K=\varnothing$ for $\delta$ less than some $\delta_{0}$. For any $\delta<\delta_{0}$, I have that $\int_{K}\left|D \chi_{B \delta}\right| \leqq \int_{K}\left|D \chi_{B}\right|$. This follows from the fact that the plane $u=\delta$ is a minimal surface, thus $\{u=\delta\} \cap B$ is the surface of smallest area with boundary $\{u=\delta\} \cap \partial B$. But for $\delta$ sufficiently small,

$$
\mu_{n}(A \cap K \cap\{u<\delta\})<\frac{\varepsilon}{\rho g},
$$

so that changing from $B$ to $B^{\delta}$ only increases the potential energy due to gravity by a small amount, while actually lowering the perimeter in $K$. So, for sufficiently small $\delta, B^{\delta}$ is the desired $\hat{B}$, proving the lemma. 
The problem of asymmetric perturbations is handled by the following theorem.

THEOREM 5.2. If a symmetric liquid bridge lifted by a disc is an energy minimum over symmetric compact perturbations, it is also an energy minimum over nonsymmetric compact perturbations.

Proof. Let $\Omega$ be the symmetric liquid bridge lifted by a disc $\mathscr{D}$ at height $h$, and suppose $\Omega_{1}$ to be a compact perturbation with $\left(\Omega \Delta \Omega_{1}\right) \subseteq K$, and $\mathscr{E}_{K}\left(\Omega_{1}\right)<\mathscr{E}_{K}(\Omega)$. By enlarging $K$, I may assume that it is symmetric about the $x_{n}$ axis.

From Lemma 5.1, there is a set $\hat{\Omega}_{1}$ and a $\delta>0$ so that $\left(\hat{\Omega}_{1} \Delta \Omega\right) \subseteq$ $K \cap\{u \geqq \delta\}$ and

$$
\mathscr{E}_{K}\left(\hat{\Omega}_{1}\right)<\mathscr{E}_{K}(\Omega)
$$

Let $\hat{\Omega}_{1}^{s}$ be the symmetrization of $\hat{\Omega}_{1}$ as described in Gonzalez [7]. Then $\hat{\Omega}_{1}^{s}=\Omega$ below height $u=\delta$, so that $\hat{\Omega}_{1}^{s}$ will be a compact perturbation of $\Omega$. Hence, since $K$ is symmetric,

$$
\mathscr{E}_{K}\left(\hat{\Omega}_{1}^{s}\right) \leqq \mathscr{E}_{K}\left(\hat{\Omega}_{1}\right)<\mathscr{E}_{K}(\Omega) \text {. }
$$

The result now follows, since $\hat{\Omega}_{1}^{s}$ is a compact symmetric perturbation.

REMARK 5.3. The following is also true: if $\Omega$ is stable over sufficiently small symmetric compact perturbations, then it is stable over any sufficiently small compact perturbations. The proof is by the same argument as above, and noting that if $\partial \Omega_{1}$ in uniformly within some $\varepsilon$ of $\partial \Omega$, then so is $\partial \hat{\Omega}_{1}^{s}$.

REMARK 5.4. A similar result is proved by Wente [14] for pendent drops in a completely different manner. The above proof can also be made to yield Wente's result. In the case of the pendent drop, $\Omega_{1}$ can be symmetrized directly, rather than going through $\hat{\Omega}_{1}$, since it is not necessary to avoid noncompact perturbations.

Let $Y$ be the open set in the $(r, u)$ plane between the envelope and the $r$ axis. I will show that the profiles $\left\{\Gamma_{o}\right\}$ considered from $\dot{\psi}=\hat{\psi}(\sigma)$ to $\dot{\psi}=\pi$, and their slopes, form a field of extremals (see e.g., Bliss [2]) in $Y$. I will again be treating the profile curves as graphs of functions of height. The functions $r(u ; \sigma)$ satisfy equation (1.7) and the initial condition $r^{\prime}(T(\sigma) ; \sigma)=0$. Let $q(r, u)$ be defined on $Y$ by

$$
q\left(r_{0}, u_{0}\right)=\left.\frac{d r}{d u}\right|_{u_{0}}
$$

for that unique curve passing through $\left(r_{0}, u_{0}\right)$ and touching the 
envelope above $u_{0}$. Note that $q(r, u)=1 / p(r, u)$ where the latter function is defined.

Lemma 5.5. Assuming 4.7, the slope function $q(r, u)$ is $C^{1}$ in $r$ and $u$ in the interior of $Y$.

Proof. The Jacobian $J$ of the $C^{1}$ map from $(\psi, \sigma)$ to $(r(\psi ; \sigma)$, $u(\psi ; \sigma))$ is:

$$
J=\dot{u} \frac{d r}{d \psi}-\dot{r} \frac{d u}{d \psi}=\frac{r}{r u+\sin \psi}(\dot{r} \sin \psi-\dot{u} \cos \psi) .
$$

By Theorem 4.4, this is nonzero for $\hat{\psi}(\sigma)<\psi<\pi$, since the zeros of $J$ determine the envelope. Restricting attention to $\psi \in(\hat{\psi}(\sigma), \pi)$, from Theorem $4.9 \mathrm{I}$ know that $Y$ is singly covered by the profiles. Hence I can invert the map and find $\psi(r, u)$ and $\sigma(r, u)$ as $C^{1}$ functions of $r$ and $u$. It is not difficult to see that:

$$
q(r, u)=\cot \psi(r, u) .
$$

Therefore, $q$ will be $C^{1}$ in $r$ and $u$ if $\sin \psi \neq 0$. But this is indeed the case, since $\psi<\pi$ on all profile curves, and $0<\hat{\psi}(\sigma)$ by Theorem 4.4 .

THEOREM 5.6. The one-parameter family of profile curves $r(u ; \sigma)$, restricted to the part of the profile with $\psi>\hat{\psi}(\sigma)$, form a field of extremals for the energy functional

$$
I(f)=\int\left(2 \pi f \sqrt{1+\left(f^{\prime}\right)^{2}}+\pi u f^{2}\right) d u \equiv \int F\left(u, f, f^{\prime}\right) d u
$$

in the region $Y$.

Proof. All that is needed for this is that $Y$ is simply covered, and that the slope function $q(r ; u)$ is $C^{1}$, proven in Lemma 5.5. See Sagan [12].

LEMMA 5.7. The Weierstrass $E$ function

$$
E\left(u, r, r^{\prime}, R^{\prime}\right) \equiv F\left(u, r, R^{\prime}\right)-F\left(u, r, r^{\prime}\right)-\left(R^{\prime}-r^{\prime}\right) F_{r^{\prime}}\left(u, r, r^{\prime}\right)
$$

is strictly positive if $r^{\prime} \neq R^{\prime}$.

Proof. $E\left(u, r, r^{\prime}, R^{\prime}\right)=\left(2 \pi r / \sqrt{1+\left(r^{\prime}\right)^{2}}\right)\left\{\sqrt{1+\left(R^{\prime}\right)^{2}} \sqrt{1+\left(r^{\prime}\right)^{2}}-\right.$ $\left.\left(1+R^{\prime} r^{\prime}\right)\right\}$. Writing this as:

$$
\begin{aligned}
E\left(u, r, r^{\prime}, R^{\prime}\right)= & \frac{2 \pi r}{\sqrt{1+\left(r^{\prime}\right)^{2}}}\left\{\sqrt{1+\left(R^{\prime}\right)^{2}+\left(r^{\prime}\right)^{2}+\left(R^{\prime} r^{\prime}\right)^{2}}\right. \\
& \left.-\sqrt{1+2 r^{\prime} R^{\prime}+\left(R^{\prime} r^{\prime}\right)^{2}}\right\}
\end{aligned}
$$


the result follows.

THEOREM 5.8. Fix an extremal $r(u ; \sigma)$, where as before I restrict my attention to that part of $\Gamma_{\sigma}$ with $\hat{\psi}(\sigma)<\psi<\pi$ (so that the domain of $r(u ; \sigma)$ is restricted to $(0, u(\hat{\psi}(\sigma) ; \sigma)))$. Pick $u_{1}, u_{2} \in$ $(0, u(\hat{\psi}(\sigma) ; \sigma))$ with $u_{2}>u_{1}$. Let $y(u)$ be a differentiable function on $\left[u_{1}, u_{2}\right]$ with $y\left(u_{1}\right)=r\left(u_{1} ; \sigma\right), y\left(u_{2}\right)=r\left(u_{2} ; \sigma\right)$, and the graph of $y$ contained in $Y$. Then

$$
\int_{u 1}^{u_{2}} F\left(u, r, r^{\prime}\right) d u \leqq \int_{u_{1}}^{u_{2}} F\left(u, y, y^{\prime}\right) d u
$$

with strict inequality holding in (5.1) unless $r(u ; \sigma)=y(u)$ for $u \epsilon$ $\left[u_{1}, u_{2}\right]$.

Proof. This follows from well-known properties of fields of extremals. The statement about strictness of inequality (5.1) comes from Lemma 5.7, since

$$
I(y)-I(r)=\int_{u_{1}}^{u_{2}} E\left(u, r, q, y^{\prime}\right) d u,
$$

and $y^{\prime}=q$ on $\left[u_{1}, u_{2}\right]$ would imply that $y(u)=r(u)$ from the definition of $q$.

COROLlaRY 5.9. The bridge surface obtained by rotating the profile curve $(r(\psi ; \sigma), u(\psi ; \sigma))$ with $\psi \in(\hat{\psi}(\sigma), \pi)$ is energy minimizing over smooth compact perturbations which are contained in the solid obtained by rotating $Y$.

Proof. This follows from Theorems 5.8 and 5.2, and noting that the symmetrization procedure (see Gonzalez [7]) yields not only a symmetric surface, but one with the property that the radius is a (single-valued) function of the axis of symmetry.

To go along with the stability result of Theorem 5.8, there is a companion instability theorem which states that if a profile curve touches the envelope, it is unstable. To demonstrate this, I need the following technical lemmas.

Lemma 5.10. Suppose that $r(u) \in C^{1}(0, h]$ has the following properties:

(i) $r^{\prime}(u)<0$ in a neighborhood of zero.

(ii) $r \in L^{2}(0, h]$.

(iii) On the interval $[\delta, h], \delta>0, r$ minimizes $I(r)$ (see Theorem 5.6) over perturbations leaving $r(h)$ and $r(\delta)$ fixed. 
Then $r(u)$ minimizes

$$
\widetilde{I}(f)=\int_{0}^{h} 2 \pi f\left(f^{\prime}+\sqrt{1+\left(f^{\prime}\right)^{2}}\right)+\pi u f^{2} d u
$$

over the set of perturbations leaving $r(h)$ fixed and $r$ fixed in a neighborhood of zero.

Proof. Since $0<r^{\prime}+\sqrt{1+\left(r^{\prime}\right)^{2}} \leqq 1$ in a neighborhood of zero by part (i), I can conclude immediately that $\widetilde{I}(r)<\infty$ by part (ii) and the Schwarz inequality. Now, on any interval $[\delta, h], I(r)$ and $\widetilde{I}(r)$ differ by the constant $\pi\left(r^{2}(h)-r^{2}(\delta)\right)$ which will remain the same after any perturbation leaving $r(h)$ and $r(\delta)$ fixed. The result follows.

LEMMA 5.11. Suppose that on $(0, h]$ there are two positive functions $r_{1}(u)$ and $r_{2}(u)$ in $C^{1}(0, h]$ with:

(i) $r_{1}^{\prime}(u)<0, r_{2}^{\prime}(u)<0$ for $u$ in a neighborhood of zero,

(ii) $r_{1}, r_{2} \in L^{2}(0, h]$

(iii) $\widetilde{I}\left(r_{1}\right)<\widetilde{I}\left(r_{2}\right)$

(iv) $r_{1}(h)=r_{2}(h)$

(v) $r_{1}(u)<r_{2}(u)$ in a neighborhood of zero.

Then a function $r_{3}(u)$ can be constructed with $r_{3}(h)=r_{2}(h)$, $r_{3}(u)=r_{2}(u)$ in a neighborhood of zero, and $\widetilde{I}\left(r_{3}\right)<\widetilde{I}\left(r_{2}\right)$.

Proof. Let $\varepsilon=\widetilde{I}\left(r_{2}\right)-\widetilde{I}\left(r_{1}\right)$, and pick $\delta \in(0,1)$ so that $\int_{0}^{\delta} r_{1}^{2}<$ $\varepsilon / 16 \pi, \int_{0}^{\delta} r_{2}^{2}<\varepsilon / 16 \pi$, and $r_{1}^{\prime}$ and $r_{2}^{\prime}$ are negative on $(0, \delta]$. Let $\phi$ be a $C^{\infty}$ nondecreasing function with $\phi \equiv 0$ on $[0, \delta / 2]$ and $\phi \equiv 1$ on $[\delta,+\infty)$. Define $r_{3}$ by:

$$
r_{3}(u)=\phi(u) r_{1}(u)+(1-\phi(u)) r_{2}(u) \text {. }
$$

Then,

$$
\begin{aligned}
\widetilde{I}\left(r_{3}\right)= & \int_{\delta}^{h} 2 \pi r_{1}\left(r_{1}^{\prime}+\sqrt{1+\left(r_{1}^{\prime}\right)^{2}}\right)+\pi u r_{1}^{2} d u \\
& +\int_{\delta / 2}^{\delta} 2 \pi r_{3}\left(r_{3}^{\prime}+\sqrt{1+\left(r_{3}^{\prime}\right)^{2}}\right)+\pi u r_{3}^{2} d u \\
& +\int_{0}^{\delta / 2} 2 \pi r_{2}\left(r_{2}^{\prime}+\sqrt{1+\left(r_{2}^{\prime}\right)^{2}}\right)+\pi u r_{2}^{2} d u .
\end{aligned}
$$

From assumption (v) and the definition of $r_{3}$, it follows that $r_{3}^{\prime}(u)<0$ on $(0, \delta]$, thus

$$
0<r_{3}^{\prime}+\sqrt{1+\left(r_{3}^{\prime}\right)^{2}}<1 .
$$

I may therefore write: 


$$
\begin{gathered}
\widetilde{I}\left(r_{3}\right)<\int_{\delta}^{h} 2 \pi r_{1}\left(r_{1}^{\prime}+\sqrt{1+\left(r_{1}^{\prime}\right)^{2}}\right)+\pi u r_{1}^{2} d u \\
+2 \pi \max \left(\int_{\delta / 2}^{\delta}\left|r_{1}\right|, \int_{\delta / 2}^{\delta}\left|r_{2}\right|\right) \\
+\pi \delta \max \left(\int_{\delta / 2}^{\delta}\left|r_{1}\right|^{2}, \int_{\delta / 2}^{\delta}\left|r_{2}\right|^{2}\right) \\
\quad+2 \pi \int_{0}^{\delta / 2}\left|r_{2}\right|+\pi \delta \int_{0}^{\delta / 2}\left|r_{2}\right|^{2} .
\end{gathered}
$$

By Schwarz's inequality,

$$
\int_{0}^{\delta} f \leqq \int_{0}^{\delta} f^{2} \cdot \delta \leqq \int_{0}^{\delta} f^{2}
$$

so by the choice of $\delta$, it follows that

$$
\widetilde{I}\left(r_{3}\right)<\int_{\delta}^{h} 2 \pi r_{1}\left(r_{1}^{\prime}+\sqrt{1+\left(r_{1}^{\prime}\right)^{2}}\right)+\pi u r_{1}^{2} d u+\frac{\varepsilon}{2} .
$$

Since $r_{1}$ is positive, I have that:

$$
\widetilde{I}\left(r_{3}\right)<\widetilde{I}\left(r_{1}\right)+\frac{\varepsilon}{2} \leqq \widetilde{I}\left(r_{2}\right),
$$

and $r_{2}$ also fulfills the other requirements laid out above.

The above two lemmas where inspired by Gerhardt's variational approach to the exterior problem [5], although they yield a much weaker result. The difference is that Gerhardt was able to consider his surfaces as graphs over the base plane, whereas I am forced to work with functions of height.

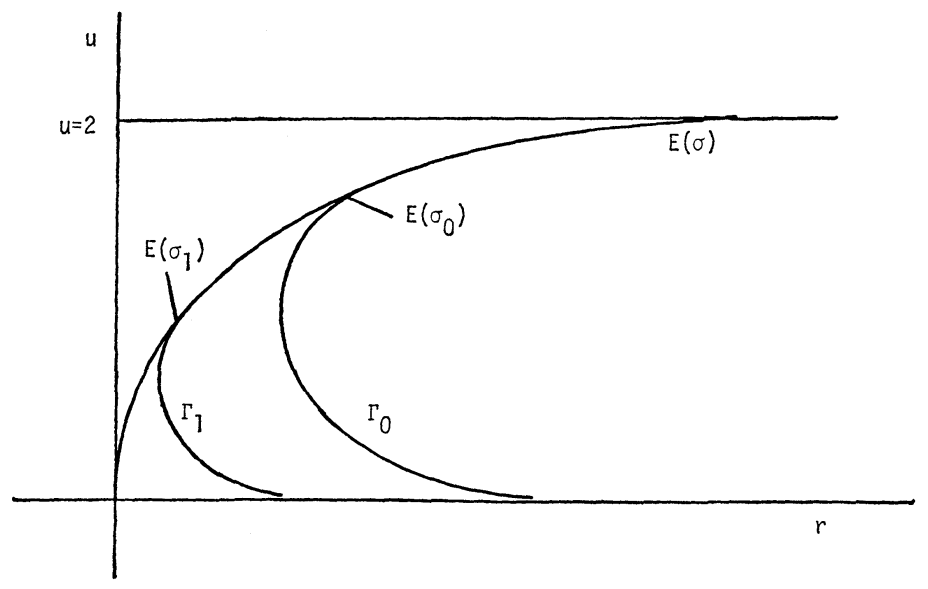

FIGURE 10

Lemma 5.12 
LeMma 5.12. Let $E\left(\sigma_{0}\right)=\left(r\left(\hat{\psi}\left(\sigma_{0}\right) ; \sigma_{0}\right), u\left(\hat{\psi}\left(\sigma_{0}\right) ; \sigma_{0}\right)\right)$ be a point on the envelope. Let $\sigma_{1} \in\left(0, \sigma_{0}\right)$. Let $C=\left\{E(\sigma) ; \sigma_{1}<\sigma<\sigma_{0}\right\} \cup\left\{\left(r\left(\psi ; \sigma_{1}\right)\right.\right.$, $\left.\left.u\left(\psi ; \sigma_{1}\right)\right) ; \psi \in\left(\hat{\psi}\left(\sigma_{1}\right), \pi\right)\right\}$ (see Figure 10$)$. Then, abusing notation slightly, $\widetilde{I}\left(\Gamma_{o}\right)=\widetilde{I}(C)$ on the interval $\left(0, u\left(\hat{\psi}\left(\sigma_{0}\right) ; \sigma_{0}\right)\right]$.

Proof. This is essentially the same as the envelope theorem (see Bliss [2]). The one difference is that I do not have a central field of extremals, i.e., a field emanating from one point. I must use a limiting argument. Pick a radius $R$, and let $p_{2}$ and $p_{3}$ be the intersections of the line $r=R$ with $\Gamma_{0}$ and $\Gamma_{1}$ (corresponding to $\sigma_{0}$ and $\sigma_{1}$ ) respectively. Following Bliss, let $\Gamma_{02}$ be the part of $\Gamma_{0}$ from $E\left(\sigma_{0}\right)$ to $p_{2}$, let $\Gamma_{13}$ be the curve $\Gamma_{1}$ from $E\left(\sigma_{1}\right)$ to $p_{3}$, let $C_{01}$ be the envelope from $E\left(\sigma_{0}\right)$ to $E\left(\sigma_{1}\right)$ and let $C_{23}$ be the segment connecting $p_{2}$ and $p_{3}$. The Hilbert invariant integral $\widetilde{I}^{*}$ is

$$
\begin{aligned}
\tilde{I}^{*}(f)=\int & 2 \pi f\left(q+\sqrt{1+q^{2}}\right)+\pi u f^{2} \\
& +\left(f^{\prime}-q\right) 2 \pi f\left(1+\frac{q}{\sqrt{1+q^{2}}}\right) d u
\end{aligned}
$$

for a function $f(u)$ (see Bliss [2]). I have:

$$
\widetilde{I}\left(\Gamma_{02}\right)-\widetilde{I}\left(\Gamma_{13}\right)=\widetilde{I}^{*}\left(C_{01}\right)-\widetilde{I}^{*}\left(C_{23}\right)
$$

(again abusing notation). It is clear from (5.2) that $\widetilde{I}^{*}\left(C_{01}\right)=\widetilde{I}\left(C_{01}\right)$, since on the envelope, the second term in the integral is zero. What must therefore be done is to show that as $R \rightarrow \infty, \widetilde{I}^{*}\left(C_{23}\right)$ can be made as small as described.

$$
\begin{aligned}
\widetilde{I}^{*}\left(C_{23}\right)= & \int_{u_{3}}^{u_{2}} 2 \pi R\left(q+\sqrt{1+q^{2}}\right)+\pi u R^{2} \\
& -2 \pi R q\left(1+\frac{q}{\sqrt{1+q^{2}}}\right) d u \\
= & \int_{u_{3}}^{u_{2}} 2 \pi R\left(\frac{1}{\sqrt{1+q^{2}}}\right)+\pi u R^{2} d u,
\end{aligned}
$$

where $u_{2}$ and $u_{3}$ are the heights of $p_{2}$ and $p_{3}$ respectively. First, to prove that $\int_{u_{3}}^{u_{2}} R / \sqrt{1+q^{2}} d u$ approaches zero. On the segment $C_{2 \mathrm{a}}$ there holds:

$$
q(u, R) \leqq q\left(u_{2}, R\right) \leqq 0 \text {. }
$$

Since $q\left(u_{2}, R\right)=r^{\prime}\left(u_{2} ; \sigma_{0}\right)$, it will suffice to show that for fixed $\sigma_{0}$, $r\left(u_{2} ; \sigma_{0}\right) / r^{\prime}\left(u_{2} ; \sigma_{0}\right)$ approaches zero as $u_{2}$ goes to zero. But this was seen in the proof of Theorem 1.1. 
Now I must show that $\int_{u_{3}}^{u_{2}} u R^{2} d u$ approaches zero. But

$$
\int_{u_{3}}^{u_{2}} u R^{2} d u=\frac{R^{2}}{2}\left(u_{2}^{2}-u_{3}^{2}\right)<\frac{R^{2} u_{2}^{2}}{2}
$$

From Siegel's asymptotic estimates [13], I know that $u_{2} \sim C e^{-R} / \sqrt{R}$, so that this term also approaches zero. Thus as $R \rightarrow \infty, \widetilde{I}^{*}\left(C_{23}\right) \rightarrow 0$.

From this fact and equation (5.3), I have

$$
\lim _{R \rightarrow+\infty}\left(\widetilde{I}\left(\Gamma_{02}\right)-\widetilde{I}\left(\Gamma_{13}\right)\right)=\widetilde{I}\left(C_{01}\right) .
$$

By the Lebesgue dominated convergence theorem, the left hand side of (5.5) is approaching

$$
\widetilde{I}\left(\Gamma_{0} \cap\left\{u \leqq u\left(\hat{\psi}\left(\sigma_{0}\right) ; \sigma_{0}\right)\right\}\right)-\widetilde{I}\left(\Gamma_{1} \cap\left\{u \leqq u\left(\hat{\psi}\left(\sigma_{1}\right) ; \sigma_{1}\right)\right\}\right) .
$$

This yields the desired result, since $C=C_{01} \cup\left(\Gamma_{1} \cap\left\{u \leqq u\left(\hat{\psi}\left(\sigma_{1}\right) ; \sigma_{1}\right)\right\}\right)$.

REMARK 5.13. The envelope does not satisfy differential equation (1.7). Indeed, the envelope has mean curvature approaching zero at large radii, but its height does not go to zero. It can also be shown to behave incorrectly for small radii. This is in contrast with the fact that the envelope of a family of curves satisfying a first order differential equation is a singular solution of that equation.

LEMMA 5.14. Given the curve $C$ in Lemma 5.12, there is a perturbation $\widetilde{C}$ which leaves $C$ fixed at $h$ and in a neighborhood of zero, and

$$
\widetilde{I}(\widetilde{C})<\widetilde{I}(C)
$$

Proof. Pick two points $P$ and $Q$ on the envelope between $E\left(\sigma_{0}\right)$ and $E\left(\sigma_{1}\right)$. Since the envelope does not satisfy the Euler equations (Remark 5.13), it follows that there is a curve $\gamma$ which is the graph of a function of height connecting $P$ and $Q$, with

$$
\widetilde{I}(\gamma)<\widetilde{I}\left(E_{P Q}\right)
$$

(Here $E_{P Q}$ is the part of the envelope between $P$ and Q.) Replacing $E_{P Q}$ by $\gamma$ gives the desired result.

THEOREM 5.15 (Instability). In the symmetric liquid bridge problem for a disc of radius $R$ at height $h$, if a profile curve goes through the point $(R, h)$, and touches the envelope at a height less than or equal to $h$, then it represents a surface which is unstable under compact perturbations. 
Proof. This is shown by putting together Lemmas 5.10 through 5.14. Given a curve $\Gamma_{0}$ which touches the envelope, I can construct the curve $C$ in Lemma 5.12 to have the same $\widetilde{I}$ energy, and perturb $C$ as in Lemma 5.14 to get $\widetilde{C}$, with $\widetilde{I}(\widetilde{C})<\widetilde{I}\left(\Gamma_{0}\right)$ on $(0, h]$. Now $\widetilde{C}$ and $\Gamma_{0}$ agree at height $h$, but not in a neighborhood of zero. However, $\widetilde{C}$ and $\Gamma_{0}$ satisfy the hypotheses of Lemma 5.11 , so that $\widetilde{C}$ can be perturbed to obtain $C^{*}$ with $\widetilde{I}\left(C^{*}\right)<\widetilde{I}\left(\Gamma_{0}\right)$, and $C^{*}$ equalling $\Gamma_{0}$ in a neighborhood of zero. From Lemma 5.10, I then conclude that $\Gamma_{0}$ does not minimize $I$ over compact perturbations, as I wished to show. In the construction of $C, \sigma_{1}$ can be chosen as close to $\sigma_{0}$ as desired, showing that $\Gamma_{0}$ is unstable even under small perturbations.

REMARK 5.16. Combining the above theorem with Corollary 5.9, Theorem 4.8 and Theorem 4.9, I can make the following statements for the completely wetting case $\beta=1$ : For a disc of radius $R$ at height $h>0$, if $(R, h)$ is outside of the region $Y$ (this includes the possibility that $(R, h)$ is on the envelope), there is no stable unbounded symmetric liquid bridge. If $(R, h)$ is contained in $Y$, there is precisely one stable profile through that point, and there is at most one unstable profile through $(R, h)$. (Recall that $Y$ is the open region between the envelope and the $r$ axis.)

REMARK 5.17. I can also characterize the stability of liquid bridges for $0 \leqq \beta<1$. It is still true that if $(R, h) \notin Y$, there can be no stable symmetric bridge. Let $\bar{\psi}$ be the inclination, at $(R, h)$, of the profile curves through $(R, h)$ which touches the envelope $h$. From inequality (1.6), if $\cos \bar{\psi} \geqq \beta$, then the bridge is unstable. If $\cos \bar{\psi}<\beta$, then the entire disc is wetted, so the stability criteria are the same as in the case $\beta=1$.

I would like to thank Prof. Robert Finn for all his help in this reserch. My thanks also to Nicholas Korevaar, Frédéric Brulois, Henry Wente, and Claus Gerhardt for helpful conversations.

\section{REFERENCES}

1. G. Bakker, Kapillarität und Oberflächenspannung. Handbuch der Experimentalphysik, vol. VI (ed. W. Wien \& F. Harms). Leipzig: Akademische Verlagsgesellschaft (1928).

2. G. A. Bliss, Lectures on the Calculus of Variations, UnIversity of Chicago Press, Chicago, 1946.

3. P. Concus and R. Finn, The shape of a pendent liquid drop, Phil. Trans. R. Soc. Lond. A292 (1979): 307-340.

4. R. Finn, The sessile liquid drop, I. Symmetric case, Pacific J. Math., 88 (1980), 541-587.

5. C. Gerhardt, A further note on the exterior capillarity problem, Math. Z., 150 
(1976), 149-157.

6. E. Gonzalez, Regolarità per il problema della goccia appoggiata, Rend. Sem. Mat. Univ. Padova, 58 (1977), 24-33.

7. — Sul problema della goccia appoggiata, Rend. Sem. Mat. Univ. Padova, 55 (1976), 289-301.

8. W. E. Johnson and L. M. Perko, Interior and exterior boundary value problems from the theory of the capillary tube, Arch. Rat. Mech. Anal., 29 (1968), 125-143.

9. P. S. Laplace, Traité de Mechanique Céleste, vol. 4, supplement to Book V, GauthierVillars, 1805.

10. I. G. Petrovsky, Ordinary Differential Equations, trans. by R. A. Silverman, Prentice-Hall, Englewood Cliffs, N. J., 1966.

11. E. Pitts, The stability of a meniscus joining a vertical rod to a bath of liquid, J. Fluid Mech., 76 (1976), 641-651.

12. H. Sagan, Introduction to the Calculus of Variations, McGraw-Hill, New York, 1969.

13. D. Siegel, Height estimates for capillary surfaces, Doctoral Dissertation, Stanford University, 1978. Pacific J. Math., 88 (1980), 471-515.

14. B. Turkington, Height estimates for capillary-type problems, Pacific J. Math., 88 (1981), 517-540.

15. H. Wente, The stability of the axially symmetric pendent drop, Pacific J. Math.,

88 (1981), 421-470.

Received April 13, 1981. The research for this paper was done when the author was at Stanford University.

NoRTHWESTERN UNIVERSITY

Evanston, IL 60201 



\section{PACIFIC JOURNAL OF MATHEMATICS}

\section{EDITORS}

DONALD BABBITT (Managing Editor)

University of California

Los Angeles, California 90024

Hugo RossI

University of Utah

Salt Lake City, UT 84112

C. C. Moore and Arthur Agus

University of California

Berkeley, CA 94720
J. DugundJI

Department of Mathematics University of Southern California Los Angeles, California 90007

R. FInN and J. Milgram Stanford University Stanford, California 94305

ASSOCIATE EDITORS
R. ARNES
E. F. BeCKenbach
B. H. NeumanN
F. WOLF
K. YoSHIDA

\section{SUPPORTING INSTITUTIONS}

UNIVERSITY OF ARIZONA

UNIVERSITY OF BRITISH COLUMBIA

CALIFORNIA INSTITUTE OF TECHNOLOGY

UNIVERSITY OF CALIFORNIA

MONTANA STATE UNIVERSITY

UNIVERSITY OF NEVADA, RENO

NEW MEXICO STATE UNIVERSITY

OREGON STATE UNIVERSITY
UNIVERSITY OF OREGON

UNIVERSITY OF SOUTHERN CALIFORNIA

STANFORD UNIVERSITY

UNIVERSITY OF HAWAII

UNIVERSITY OF TOKYO

UNIVERSITY OF UTAH

WASHINGTON STATE UNIVERSITY

UNIVERSITY OF WASHINGTON 


\section{Pacific Journal of Mathematics}

\section{Vol. 103, No. $1 \quad$ March, 1982}

Abdul Aziz, On the zeros of composite polynomials ..................

Salomon Benzaquen and Enrique M. Cabaña, The expected measure of the level sets of a regular stationary Gaussian process $\ldots \ldots \ldots \ldots \ldots$

Claudio D’Antoni, Roberto Longo and László Zsidó, A spectral mapping theorem for locally compact groups of operators $\ldots \ldots \ldots \ldots \ldots \ldots \ldots 17$

Ronald Dotzel, Semifree finite group actions on homotopy spheres ........ 25

Daniel H. Gottlieb, The Lefschetz number and Borsuk-Ulam theorems . . . . . 29

Shui-Hung Hou, On property $(Q)$ and other semicontinuity properties of

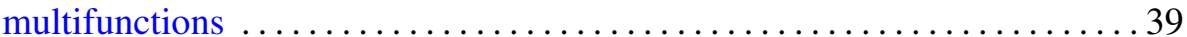

Kevin Mor McCrimmon, Compatible Peirce decompositions of Jordan triple systems

Mitsuru Nakai, Corona problem for Riemann surfaces of Parreau-Widom

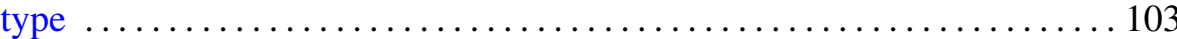

Jack Ray Porter and R. Grant Woods, Extensions of Hausdorff spaces . . . . 111

Milton Rosenberg, Quasi-isometric dilations of operator-valued measures

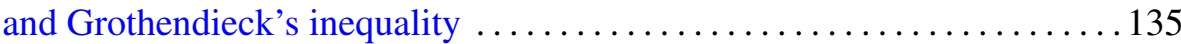

Joseph L. Taylor, A bigger Brauer group $\ldots \ldots \ldots \ldots \ldots \ldots \ldots \ldots \ldots \ldots$

Thomas Vogel, Symmetric unbounded liquid bridges . . . . . . . . . . . 205

Steve Wright, The splitting of operator algebras. II ............... 243 